\title{
Ludwig Erhards Konzept der sozialen Marktwirtschaft und seine wettbewerbstheoretischen Grundlagen
}

Martin Rhonheimer

Received 27 June 2016; Accepted 13 February 2017

Zusammenfassung: Erhards Konzeption einer sozialen Marktwirtschaft durch marktwirtschaftlichen Wettbewerb ist auch heute noch wegweisend. Doch wurde die Idee des Sozialen im Programm der Sozialen Marktwirtschaft mit der Zeit verfälscht. Zudem beruht Erhards Konzept auf einer wettbewerbstheoretischen Grundlage - dem neoklassischen Modell der vollständigen Konkurrenz -, die seinen wohlstandsfördernden Intentionen zuwiderläuft. Erhards Position stimmte mit derjenigen der Freiburger Schule (Walter Eucken, Franz Böhm) nur teilweise überein, unterscheidet sich aber ebenso in wesentlichen Punkten vom Denken der Österreichischen Schule der Nationalökonomie (Ludwig von Mises, Friedrich August Hayek) und deren dynamisch-evolutionärem Verständnis von Wettbewerb. Gerade für eine im Sinne Erhards wettbewerbsorientierte Konzeption der Sozialen Marktwirtschaft erscheint das Österreichische Verständnis von Wettbewerb als das realitätsnähere und adäquatere.

Schlagwörter: $\quad$ Soziale Marktwirtschaft $\bullet$ Wettbewerb $\bullet$ Sozialstaat $・$ neoklassische Wettbewerbstheorie

(c) De Gruyter Open Sp. z 0.0 .

\section{Einleitung}

Ludwig Erhards Buch „Wohlstand für alle“ aus dem Jahr 1957 ist für den heutigen Leser eine Lektüre voller Überraschungen. Er entdeckt, dass für den Begründer der sozialen Marktwirtschaft das Soziale an der sozialen Marktwirtschaft Markt und Wettbewerb sind, und nicht eine die Marktwirtschaft ergänzende oder ihre Ergebnisse korrigierende Sozialpolitik. Dieses Konzept einer Schaffung von Wohlstand durch Markt und Wettbewerb und die damit verbundene Ablehnung des umverteilenden Versorgungs- und Wohlfahrtsstaates entspricht jedoch nicht der heutzutage gängigen Vorstellung von Sozialer Marktwirtschaft. Diese entwickelte sich erst nach und nach aus den Ansätzen eines anderen
Konzepts, das bereits den Keim der heutigen sozialstaatlichen Umverteilungshypertrophie in sich trug.

Meiner Ansicht nach ist Erhards Konzeption einer sozialen Marktwirtschaft durch Entfesselung des marktwirtschaftlichen Wettbewerbs auch heute noch wegweisend. Doch wurde die Idee des Sozialen im Programm der sozialen Marktwirtschaft - nicht ohne Mitschuld ihrer Begründer - mit der Zeit verfälscht. Das Konzept der sozialen Marktwirtschaft beruht zudem auf einer wettbewerbstheoretischen Grundlage - dem neoklassischen Modell der vollständigen Konkurrenz -, die seinen wohlstandsfördernden Intentionen zuwiderläuft. Um dies näher zu begründen, soll im Folgenden erstens Erhards Konzeption der sozialen Marktwirtschaft als 
wohlstandsschaffende Wettbewerbsordnung und deren spätere Verfälschungen durch den überbordenden Sozialstaat dargestellt werden. Es geht dabei auch um die Frage, wie weit diese Entwicklung nicht bereits im anfänglichen Konzept (vor allem Müller-Armacks) angelegt war.

Zweitens werde ich auf die wettbewerbstheoretischen Grundlagen von Erhards Konzeption eingehen: das neoklassische Gleichgewichtsmodell der vollständigen Konkurrenz und Erhards damit begründetes Eintreten für eine konsequente Antikartell- und Antimonopolpolitik. Dabei wird sich auch die Frage stellen, in welchem Maß seine Vorstellungen mit denen der Freiburger Schule (Ordoliberalismus) übereinstimmen. Schließlich werde ich das dem Denken der Österreichischen Schule der Nationalökonomie entstammende dynamische und evolutionäre Verständnis von Wettbewerb der ordoliberalen Antikartell- und Antimonopolpolitik gegenüberstellen und für die Überlegenheit des dynamisch-evolutionären Paradigmas von Wettbewerb plädieren. Dabei ist zu zeigen, dass dieses für die Konzeption einer sozialen Marktwirtschaft im Erhard'schen Sinn - Wohlstand durch Wettbewerb - das geeignetere und realitätsnähere wäre und angesichts der aktuellen Degenerierung der sozialen Marktwirtschaft in Richtung eines innovationshemmenden Etatismus und einer wohlstandsvernichtenden Umverteilungsmaschinerie eine echte Alternative bietet.

\section{Der moderne Sozialstaat: Hemmschuh für die Wohlstandsmehrung}

Wie aus Erhards eingangs genannten Buch hervorgeht, heißt soziale Marktwirtschaft für inn, Wohlstand für alle nicht durch eine bestimmte Art von Sozialpolitik, soziale Bändigung des freien Marktes oder Korrektur seiner distributiven Ergebnisse, sondern, eher umgekehrt, diesen allen zugutekommenden Wohlstand durch Entfesselung von Markt und Wettbewerb zu schaffen. Eine Politik des sozialen Ausgleichs in der Form sozial- oder wohlfahrtstaatlicher Umverteilung lehnte Erhard hingegen ab. Sie sei „Flucht vor der Eigenverantwortung“ (Erhard 1964, S. 248) und eine Sucht, die zu einer gesellschaftlichen Ordnung führt „in der jeder die Hand in der Tasche des anderen hat" (Ebd.). Im Jahre 1964 war das in der Einschätzung Erhards bereits eine reale Gefahr, vor der er warnte: „Die Blindheit und intellektuelle Fahrlässigkeit, mit der wir dem Versorgungs- und Wohlfahrtsstaat zusteuern, kann nur zu unserem Unheil ausschlagen“ (Ebd.).

Der heutige Durchschnittsbürger, aber auch die führenden Sozialethiker sowie Vertreter der christlichen
Gesellschaftslehre sind heute davon überzeugt, dass es ohne solche Umverteilung nicht geht, ja, dass wir in Wirklichkeit dem umverteilenden Sozialstaat unseren Wohlstand verdanken. Das Unheil, vor dem Erhard gewarnt hatte, ist also in vollem Gange. Es geht einher mit einem gravierenden Bedeutungsverlust der Familie als tragende gesellschaftliche Lebens- und Vorsorgestruktur und äußert sich in einem fast maßlosen Anspruchsdenken, das zu einer nicht nachhaltigen, stetig wachsenden öffentlichen Verschuldung geführt hat, die momentan nur noch dank der Niedrigzinspolitik der Zentralbanken tragbar ist. Die möglichen Folgen einer übermäßigen öffentlichen Verschuldung werden die nächsten Generationen zu verkraften haben. ${ }^{1}$

Die „Entwicklung zum Versorgungsstaat“ - so Erhard - sei „schon dann eingeleitet, wenn der staatliche Zwang über den Kreis der Schutzbedürftigen hinausgreift“ (Ebd., S. 251). Deshalb plädierte er gegen einen allgemeinen Versicherungszwang - gegen ein System, wie es nicht nur in Deutschland im heute real existierenden Sozialstaat schon seit langem verwirklicht ist. Er prognostizierte richtig eine „Aufblähung der öffentlichen Haushalte" (Ebd.) und zunehmende Abhängigkeit des Bürgers vom Staat - eine der Ursachen heutiger weitgehender Funktionslosigkeit der Familie. Geradezu prophetisch meinte er: „Die Sicherheit des einzelnen Menschen - oder mindestens das Sicherheitsgefühl hat mit der Überantwortung seines Schicksals an den Staat oder an das Kollektiv nicht zugenommen, sondern abgenommen" (Ebd.).

In der Tat finden wir heute ein allgemeines Lamento auf hohem Niveau - über soziale Ungleichheit, Schrumpfen der Mittelschicht, Umweltverschmutzung,

1 Große Risiken bestehen hier, abgesehen von der Verdrängung (crowding out) privater Investitionen und damit verbundener technologischer Innovation als Folge steigender Staatsschulden, vor allem wegen demographischer Veränderungen, die gerade von einem umlagefinanzierten (d.h. steuerfinanzierten) Pensionssystem hervorgerufen werden und infolge ihrer wachstumsvermindernden Auswirkungen auch die Vorschläge von Diamond und Orszag (2005), den sogenannten Brookings Plan, nicht-nachhaltig erscheinen lassen. Vgl. dazu wie auch grundlegend zur ökonomischen Anreizproblematik und entsprechenden Gerechtigkeitsfragen des Wohlfahrtsstaates Shapiro (2007); zu Diamond und Orszag Ebd., S. 289 ff. Zur unter Ökonomen umstrittenen Frage des Einflusses von Staatschulden auf die zukünftige Kapitalausstattung einer Volkswirtschaft und die Konsummöglichkeiten zukünftiger Generationen vgl. Holtfrerich; Feld, Lars P.; Heun et al. (2015), S. 36 ff. und 54 ff. (darin auch Verweise auf die Arbeit von Diamond [1965] über die Beziehung zwischen realem Zinssatz und realer Wachstumsrate im langfristigen Gleichgewicht, die anhand eines mathematischen Modells zeigt, dass höhere Staatsverschuldung, - vorausgesetzt, die durch erhöhte Verschuldung erzielte Wachstumsrate übersteigt den realen Zinssatz - das Produktionspotential späterer Generationen und deren Konsumchancen nicht verringert, wobei allerdings in diesem Modell die oben genannten Gesichtspunkte unberücksichtigt bleiben). 
Individualisierung usw. gefolgt von immer neuen Rufen nach mehr Staat, mehr Regulierung und noch mehr Absicherung. Nicht nur der einfache Bürger und Sparer, sondern auch der Unternehmer lebt in zunehmender Abhängigkeit von der Politik, insbesondere der Geldpolitik, die vom gefährlichsten aller Staatsmonopole, dem Geldmonopol, ohne klare Regeln und mit einer undurchsichtigen Agenda betrieben wird. Ein Bewusstsein hinsichtlich der wirtschaftlichen Kosten und der politischen Risiken, die in dieser Abhängigkeit liegen, besteht bei den Wenigsten. Erhards soziales Grundanliegen hatte nun aber gerade darin bestanden, durch Marktwirtschaft die Freiheit und Autonomie des Bürgers zu ermöglichen, so dass ein jeder selbstbestimmt für das eigene Leben vorzusorgen imstand ist.

Der Sozialstaat, der durch massive und progressive Besteuerung der Bürger zunehmend konsumtive Ausgaben der öffentlichen Hand finanziert, schafft weder größere Freiheitsräume und Selbstbestimmung noch Wohlstand. Er verteilt vielmehr zuvor geschaffenen Reichtum an Transferempfänger, deren Leben dadurch von staatlichen Subventionen abhängig ist. Dazu bremsen Umverteilung und Transferleistungen - aufgrund hoher Steuerlast und gerade in entwickelten Staaten die wohlstandsschaffende Dynamik der Marktwirtschaft und des für eine kapitalistische Wirtschaftsform typischen unternehmerischen Handelns. Auch die Gewerkschaften schaffen keinen Wohlstand, sondern bewirken nur seine Umverteilung, weil sie durch Hinauftreiben der Löhne Arbeitslosigkeit in anderen Sektoren der Wirtschaft erzeugen oder die Inflation antreiben, welche durch Korrosion der Reallöhne Wohlstandsgewinne zunichtemacht. Meist geschieht diese Umverteilung zu Lasten des Gemeinwohls. Allein der Produktivitätszuwachs, der immer auf Kapitalakkumulation (bzw. Sparen), Investitionen, Innovation und unternehmerischer Leistung beruht, schafft und vermehrt Wohlstand und damit auch ein Leben in Selbstbestimmung und eigener Verantwortung. Was der Staat verteilt, ist nicht Manna, das vom Himmel fällt, sondern potentiell wohlstandsmehrende Ressourcen, die dem produktiveren Teil der Gesellschaft durch Besteuerung entzogen werden, um sie dem weniger produktiven oder gänzlich unproduktiven zuzuführen. Dies setzt entsprechend falsche Signale und Anreize. Die üblen Folgen jedoch werden dem angeblich haltlos freien Markt und dem Kapitalismus angelastet.

\section{Welche soziale Marktwirtschaft?}

Erhard hatte die klare Vorstellung: Wohlstand für alle wird vom produktiven und innovativen Unternehmertum erzeugt. Aus diesem Grund hielt er die, den
Leistungswettbewerb fördernde, Marktwirtschaft selbst für eine soziale Veranstaltung und in genau diesem Sinne nannte er sie auch soziale Marktwirtschaft. Sozial war für Erhard die Marktwirtschaft allerdings nur insofern, als ihre produktiven Leistungen dem Konsumenten auch tatsächlich zugutekommen: „Das ist der soziale Sinn der Marktwirtschaft, dass jeder wirtschaftliche Erfolg, wo immer er entsteht, dass jeder Vorteil aus der Rationalisierung, jede Verbesserung der Arbeitsleistung dem Wohle des ganzen Volkes nutzbar gemacht wird und einer besseren Befriedigung des Konsums dient." (Ebd., S. 169; Hervorhebungen im Original). ${ }^{2}$ Es ist entscheidend, dass das im Auge behalten wird: Nicht um das Wohl des Produzenten geht es, auch nicht um dasjenige der Arbeitnehmer oder bestimmter Gesellschaftsgruppen, sondern um das Wohl des Konsumenten. Das sind schlicht alle Bürger, auch die Unternehmer, Produzenten, Investoren und natürlich die Arbeitnehmer: Sie alle sind Konsumenten.

Es ist geläufig, Erhard ideengeschichtlich dem Ordoliberalismus zuzurechnen. Doch entstammte er nicht der Tradition der ordoliberalen Freiburger Schule (Walter Eucken, Franz Böhm). Sein Lehrer, dem er zeitlebens ganz besonders verpflichtet blieb, war Franz Oppenheimer, der radikale Staatskritiker, der in einer freien Marktwirtschaft die Grundlage eines nichtkapitalistischen liberalen Sozialismus sah (Erhard 1964/1988, S. 861). ${ }^{3}$ Erhard kehrte den Gedanken seines Lehrers um und verfolgte die Idee eines sozialen Liberalismus durch Marktwirtschaft (Ebd.). Ebenso ist hier - neben anderen - Erhards Lehrer Wilhelm Rieger hervorzuheben, der inn zu einer konsequent privatwirtschaftlichen Sicht unternehmerischer Tätigkeit führte (Wünsche 2015, S. 286 ff.).

Schöpfer des auch von Erhard übernommenen Begriffs Soziale Marktwirtschaft ${ }^{4}$ war dessen Mitarbeiter Alfred Müller-Armack, ehemaliger Berater des NS-Regimes, anfänglicher Bewunderer seiner Arbeitsbeschaffungspolitik und seit dem Jahr 1940 Direktor des Instituts für Wirtschafts- und Sozialwissenschaften

2 Abgesehen von dem - aus Texten und Reden Erhards kompilierten und erst im Jahr 1964 von Erhard selbst überarbeiteten - Buch "Wohlstand für alle", findet sich seine wettbewerbstheoretisch begründete Konzeption der Sozialen Marktwirtschaft sehr prägnant in seiner Rede vor dem Deutschen Bundestag vom 24. März 1955 über „Die Ziele des Gesetzes gegen Wettbewerbsbeschränkungen“ (Erhard 1955/1988).

3 Dennoch trug Erhard zumindest vordergründig selbst dazu bei, den Neoliberalen der Freiburger Schule zugerechnet zu werden; vgl. z.B. Erhard 1964/1988, S. 861 (auf diesen Text werden wir noch zurückkommen).

4 Im Unterschied zu Erhard schrieb Müller-Armack Soziale Marktwirtschaft immer mit einem großen „S“, eine Schreibweise, die hier respektiert werden soll. 
der Universität Münster. Dieser verstand in einem 1946 veröffentlichten Buch die Soziale Marktwirtschaft als eine „bewusst gesteuerte, und zwar sozial gesteuerte Marktwirtschaft" (Müller-Armack 1990, S. 96). Der Irrtum des Liberalismus habe nicht nur in einem „Fehlen jeder konkreten Sicherung der marktwirtschaftlichen Form" (Ebd., S. 93) (also in reinem Laissez faire) bestanden, sondern auch in der bloß „passiven Annahme, die Ergebnisse des marktwirtschaftlichen Prozesses seien so, wie sie vorliegen, als letztgültig hinzunehmen“ (Ebd.). Doch sei durch diese Ergebnisse „die Frage der sozialen Gerechtigkeit noch nicht entschieden“ (Ebd.). Im Namen der sozialen Gerechtigkeit propagierte Müller-Armack „die Notwendigkeit eines sozialen Einkommensausgleichs" (Ebd.), Korrekturen und Ergänzungen der durch den Markt erzielten Verteilungsergebnisse (Ebd.). So entwickelte er das Konzept einer Sozialpolitik, vorwiegend durch Fiskalpolitik, die den Preismechanismus des Marktes zwar intakt hält, inn aber in den Dienst erwünschter sozialpolitischer Ziele stellt. $\mathrm{Zu}$ einem solchen Lenkungsmechanismus gehört auch „eine hohe Besteuerung zugunsten eines sozial- und staatswirtschaftlichen Konsums" (Ebd., S. 102), immer unter Beachtung der marktwirtschaftlichen Spielregeln (Ebd.). ${ }^{5}$

Schloss Müller-Armacks Konzeption von 1946 den Kern des späteren, immer mehr sozialdemokratisch geprägten Umverteilungsstaates bereits in sich? Seine Schrift aus dem Jahre 1946 ist in manchen Formulierungen reichlich ambivalent. Der SPD-nahe katholische Sozialethiker Oswald von Nell-Breuning behauptete im Jahr 1986, Müller-Armacks ursprüngliches Konzept der sozialen Marktwirtschaft, wie er es 1956 in seinem Artikel "Soziale Marktwirtschaft“ im Handwörterbuch

5 Die dieser Auffassung innewohnende Problematik zeigt sich darin, dass Müller-Armack den Markt und das Preissystem mit einer Maschine vergleicht. Die Soziale Marktwirtschaft als gesteuerte Marktwirtschaft beruht nicht auf dem Glauben, „dass es zweckmäßig ist, eine auf vollen Touren laufende Maschine sich selbst zu überlassen, wie es der Liberalismus annahm, auch nicht, dass es zweckmäßig ist, alle Ventile zuzuschrauben und alle Hebel festzuriegeln, wie es die Wirtschaftslenkung tut, sondern sie versucht, diese Maschine zu bedienen, durch ein sinnvolles Spiel von Schaltung und Sicherung, um so zu einem vernünftigen Ergebnis zu kommen" (Müller-Armack 1946/1990, S. 64). Diese Auffassung ist weit entfernt vom klassischliberalen Verständnis - und eine Karikatur desselben -, gemäß dem der Markt die Koordination einer Unzahl individueller menschlicher Entscheidungen über das Preissystem bewerkstelligt und damit zu dem Ergebnis führt, das am besten dem niemandem - auch nicht einem Wirtschaftslenker - im Voraus bekannten Gemeinwohl entspricht. Die Maschinen-Metapher Müller-Armacks ist im besten Falle unglücklich gewählt; sie lässt aber vermuten, dass ihr Autor eine falsche, nämlich mechanistische anstatt auf das menschliche Handeln und Entscheiden fokussierte Vorstellung von der klassisch-liberalen Theorie des Marktes hatte. der Sozialwissenschaften präsentierte (Müller-Armack 1956/1976), sei von der Idee einer Steuerung der Marktwirtschaft aus sozialen Gründen noch ganz frei gewesen. Als alleiniges Ziel der Steuerung habe auch er anfangs die Sicherstellung der Wettbewerbsordnung aufgefasst. Erst später sei das Konzept auch von Müller-Armack in Richtung einer sozialpolitischen Ausweitung der Wirtschaftspolitik und letztlich einer sozialdemokratischen Interpretation der sozialen Marktwirtschaft im Sinne des Godesberger Programms ausgeweitet worden (Nell-Breuning 1986/1990, S. 236 ff.).

Nell-Breunings Auslegung ist jedoch mit dem textlichen Befund nicht zu vereinbaren. Eher ist hier Horst Wünsche, dem ehemaligen Erhard-Mitarbeiter und langjährigen Geschäftsführer der Ludwig-Erhard-Stiftung, Recht zu geben, der in seiner Erhard-Monographie die Meinung vertritt, Müller-Armack habe von Anfang an eine andere, nämlich - im Gegensatz zu Erhard - eine Umverteilung befürwortende Konzeption vertreten (Wünsche 2015, S. 379 f.). Textliche Befunde sind geeignet, Wünsches Ansicht zu stützen. Zunächst stimmt es nicht, wie Nell-Breuning behauptet, dass Müller-Armack ursprünglich noch keine sozialen Gründe für Marktsteuerung anführte. Im Gegenteil: Die Soziale Marktwirtschaft, so Müller-Armack im Jahr 1956, beschränke sich nicht darauf, "das Instrumentarium der Konkurrenz sozial funktionsfähig zu machen“. Vielmehr biete der „wirtschaftliche Einkommensprozess [...] der Sozialpolitik ein tragfähiges Fundament für eine staatliche Einkommensumleitung, die in Form von Fürsorgeleistungen, Renten- und Lastenausgleichszahlungen, Wohnbauzuschüssen, Subventionen usw. die Einkommensverteilung korrigiert" (Müller-Armack 1956/1976, S. 246). ${ }^{6}$

Nicht nur das ominöse "usw." nach dem Wort "Subventionen" lässt aufhorchen (es findet sich auch in anderen, analogen Aussagen Müller-Armacks immer nach dem Wort Subventionen), sondern auch die nachfolgende Bemerkung, eine marktwirtschaftliche Sozialpolitik unterscheide sich „von der früheren lenkungswirtschaftlichen nicht durch ihre Ziele, als vielmehr durch das Instrumentale" (Ebd.). "Gewiss" - gibt Müller-Armack zu - „kann bei der Einkommensumleitung für soziale Ausgaben leicht die Schwelle überschritten werden, an der die Störung des Marktes beginnt. Wann überhöhte Steuersätze dies tun, ist nicht vorweg zu entscheiden“ (Ebd.). In einem Artikel aus dem Jahr 1947 mit dem Titel „Die Wirtschaftsordnung sozial gesehen“ plädierte Müller-Armack dafür „über

6 Ebenso schrieb er schon zehn Jahre früher: „unmittelbare Einkommensumleitung“ in der Form von „direkten Kinderbeihilfen, Mietzuschüssen, Wohnungsbauzuschüssen“ (Müller-Armack 1946/1990, S. 119). 
eine klare Einkommensbesteuerung den Ausgleich unerwünscht erscheinender Einkommensunterschiede zu bewerkstelligen“, wonach er bemerkt: „Eine solche Soziale Marktwirtschaft kann je nach dem politischen Wollen radikaler oder konservativer durchgeführt werden“ (Müller-Armack 1974, S. 84). Das Konzept ist also gleichsam nach oben hin - in Richtung von mehr Umverteilung - offen und spielt deshalb einer beständigen, durch den demokratischen Prozess unweigerlich geförderten Ausweitung staatlicher Umverteilung in die Hände. ${ }^{7}$ Seit dem Jahr 1960 schließlich sprach dann Müller-Armack folgerichtig von einer nach den ersten Erfolgen nun notwendigen zweiten Phase der Sozialen Marktwirtschaft, die sich durch eine entschiedene Ausweitung der Staatsaufgaben und staatlich angebotener Dienstleistungen charakterisieren sollte. Das Beiwort sozial in Soziale Marktwirtschaft weise ja darauf hin, dass diese Wirtschaftsordnung "gesellschaftspolitische Ziele verfolgt" (Ebd., S. 151).

Aufgrund dieses textlichen Befundes erscheint die Behauptung kaum übertrieben, mit dem von MüllerArmack im Jahr 1946 vorgestellten Konzept einer gesteuerten Marktwirtschaft durch sozialpolitische Eingriffe, die zwar die Preisbildung intakt lassen und insofern marktkonforme Eingriffe sind, sei die Büchse der sozialstaatlichen Pandora zumindest so weit geöffnet worden, dass spätere Ausweitungen im Sinne der heute real existierenden sozialen Marktwirtschaft, die staatliche Marktsteuerung mit dem schon von Müller-Armack formulierten Ziel der sozialen Gerechtigkeit betreibt und auch vor flächendeckenden Mindestlöhnen nicht mehr zurückschreckt, darin angelegt waren. Man bedenke die erstaunlichen Formulierungen in dem von Erhard und Müller-Armack gemeinsam herausgegebenen „Manifest '72". In einem der Kapitel, das sich ganz an frühere Formulierungen Müller-Armacks anlehnt, heißt es nun, die Soziale Marktwirtschaft „beseitigt die Startungleichheit auf dem Markt, die sich aus der unterschiedlichen Einkommensbildung und Eigentumsverteilung ergibt, mittels Besteuerung, Sozialversicherung und Sozialfürsorge und erreicht durch Ausbildungs- und Studienförderung, durch Prämien zur Vermögensbildung und

7 Das Konzept der sozialen Marktwirtschaft krankt zudem am Ausklammern jener Aspekte, die die Public Choice Theorie analysiert, etwa der Existenz der durch demokratische Mehrheitsentscheidungen geförderten sogenannten Umverteilungskoalitionen; vgl. Blankart (2011), S. 75: „Wählergruppen können Vorlagen zur Abstimmung bringen, die innen selbst Konsumentenrentengewinne verschaffen [...]. Doch werden die Kosten hierfür einer Wählerminderheit aufgebürdet, die ihrerseits Konsumentenrentenverluste erleidet." Dieser Prozess kann natürlich immer als sozial gerecht gerechtfertigt werden, besitzt deshalb aber naturgemäß keine Grenze nach oben; vgl. Ebd. S.96 ff.; zum Thema auch: Frey und Kirchgässner (1994), S. $210 \mathrm{ff}$.
Subventionen mittels Umverteilung einen sozialen Ausgleich" (Erhard und MüllerArmack 1972, S. 58). Hier geht es plötzlich nicht mehr um die ordoliberale Startgleichheit der im produktiven Wettbewerb stehenden Unternehmer (s. unten), sondern um Startgleichheit bezüglich des Konsums! Für ihre Herstellung sei auf Marktkonformität zu achten und es handle sich um „gezielte Eingriffe, die nur den Bedürftigen zugutekommen und keinen allgemeinen Segen bewirken“ (Ebd.). Schließlich: „Die Möglichkeit, die für die Umverteilung zur Verfügung stehende Masse zu erhöhen, liegt aber in der Ausweitung der Marktvorgänge insgesamt“ (Ebd.). Mit anderen Worten: je erfolgreicher die Marktwirtschaft, desto reicher die Gesellschaft und desto mehr kann auch umverteilt werden. Es ist ja das Sozialstaatsprinzip selbst, das der Mehrheit immer von neuem erlaubt, „die soziale Gerechtigkeit wieder neu zu definieren“ und den „Interessengruppen unbeschränkte Möglichkeiten eröffnet, Umverteilungsvorlagen zu erfinden und durchzusetzen" (Blankart 2011, S. 98). Jede Regel, auch die der sogenannten Marktkonformität kann dadurch politisch ausgehebelt werden.

So öffnet sich die Büchse der Pandora nach und nach, denn für weitere Ausweitungen des Kreises der Bedürftigen - im Dienste der Startgleichheit - und damit von Subventionierung und Umverteilung gibt es nun schlicht keine angebbaren Grenzen mehr - zumal die dadurch immer mächtiger werdende Sozialbürokratie und auf Ausweitung ihres Wählerpotentials erpichte Politiker an einer solchen Ausweitung ein offensichtliches Interesse haben. Marktkonformität ist, wie die Erfahrung zeigt, zudem ein dehnbarer Begriff und führt zur sozialpolitischen Willkür, weil Umverteilung eben keine Marktprozesse tangiert, sondern nur deren Ergebnisse korrigiert und deshalb eigentlich definitionsgemäß marktkonform ist. Solche, vielleicht aus der Situation der frühen 1970er Jahre verständliche Versuche, das Soziale an der sozialen Marktwirtschaft besonders herauszustreichen, manifestiert jedoch eine dem Konzept Müller-Armacks von Anfang an innewohnende Ambivalenz, die schließlich einen Prozess in Gang setzte - oder sich ihm zumindest nicht zu widersetzen vermochte -, der aufgrund der inhaltlichen Unbestimmtheit und Manipulierbarkeit des Begriffs soziale Gerechtigkeit den Gerechtigkeitsgedanken zunehmend korrumpieren und - unter dem Vorwand der Sozialpflichtigkeit des Eigentums - die verfassungsmäßige Eigentumsgarantie durch den Zugriff von Politik und Steuerbehörden immer mehr aushöhlen sollte. ${ }^{8}$

8 Man erinnere sich an die oben zitierten Worte Erhards aus dem Jahre 1964: „Die Blindheit und intellektuelle Fahrlässigkeit, mit der wir dem Versorgungs- und Wohlfahrtsstaat zusteuern, kann nur zu unserem Unheil ausschlagen" (Erhard 1964, S. 248). 
Zumindest bis zu seinem Ausscheiden aus der Regierungsverantwortung im Jahr 1966 war die Konzeption Erhards eine andere gewesen: Damit die Marktwirtschaft ihre soziale Aufgabe erfüllen kann, bedurfte es seiner Ansicht nach nicht der Sozialpolitik, die Marktergebnisse korrigiert, sondern einer Wettbewerbspolitik, die von Anfang an sicherstellt, dass der Markt keine konsumentenschädigende, und damit wohlstandsvermindernde, Wirkungen erzielt. Das ist in der Tat eine wesentlich eingeschränktere Konzeption, die - abgesehen von einer staatlich garantierten Fürsorge für Schutzbedürftige (so lautete Erhards Ausdruckweise) Sozialpolitik als reine Wirtschaftspolitik und diese letztlich als Wettbewerbspolitik versteht. Wettbewerb war für Erhard "das geeignete Mittel, um die soziale Schwäche der Marktpreisbildung systemgerecht aufzuheben“ (Wünsche 2015, S. 397). Die Feinde des Wettbewerbs benannte Erhard im Jahr 1955 vor dem Deutschen Bundestag in klaren Worten: es sind die Kartelle, die zu einer kollektiven Preispolitik führen, durch die diese „mehr Kaufkraft abschöpfen, als innen im freien Wettbewerb bei freien Preisen zufließen würden" (Erhard 1955/1988, S. 433). Das bewirkt Preisverzerrungen oder geringeren Absatz an anderer Stelle, schafft Ungleichgewicht auf dem Arbeitsmarkt und schädigt die Konjunktur - kurz: es schmälert den jedem Konsumenten zustehenden Teil der volkswirtschaftlichen Leistung und ist deswegen unsozial. Deshalb auch sind Kartelle gerade dann am schädlichsten, wenn sie gut funktionieren (Ebd., S. 434), und sie sind heimtückisch, weil der Konsument wegen der Komplexität des Preisbildungsprozesses ihren schädlichen Einfluss gar nicht wahrnehmen und deshalb seine Interessen auch nicht verteidigen kann (Ebd., S. 436). Deshalb ist es Aufgabe des um die wirtschaftlichen Zusammenhänge wissenden Politikers - Erhard war von diesem Ethos durchdrungen - den Konsumenten zu schützen: „Als Wirtschaftsminister aber habe ich nicht die Interessen gewerblicher Gruppen, sondern das Lebensrecht von 50 Millionen Verbrauchern zu verteidigen“ (Ebd., S. 438).

Dabei ging Erhard von einem Begriff des Leistungswettbewerbs aus, dem auch eine Freiheits- und Gerechtigkeitskonzeption zugrunde liegt: „Die ungehinderte Entfaltung der unternehmerischen Initiative hat allerdings dort eine Grenze, wo die Rechts- und Lebenssphären anderer Bevölkerungsschichten berührt werden und wo eine wirtschaftliche Position oder gar eine Machtstellung nicht mehr durch individuelle unternehmerische Leistung im Wettbewerb, sondern durch kollektive Absprachen und künstlich gesetzte Machtpositionen errungen werden will“ (Ebd., S. 435). „Freier Leistungswettbewerb" ist deshalb nur in einem Zustand gegeben, der „die Gewinnung erhöhter Erträge oder sogar Renten verhindert und die Dynamik der Wirtschaft in Gang hält" (Ebd. S., 436). Damit sind natürlich auch Monopolrenten - überhöhte, d.h. über den Grenzkosten liegende Absatzpreise von Monopolisten - gemeint, die ebenfalls ein von der Erhard'schen Politik bekämpfter Gegner waren.

In der Begründung seiner Konzeption eines wohlstandsschaffenden Wettbewerbs stand Erhard zumindest was seine Vorstellung von allokativer Ineffizienz von Monopolen und Kartellen betrifft - ganz auf dem Boden der neoklassischen Wettbewerbstheorie und Wohlfahrtsökonomik wie sie sich vor allem seit $A$. C. Pigou ${ }^{9}$ entwickelt hatte. Ihre Grundzüge finden sich heute noch in den meisten Lehrbüchern. Sie liegen in ihren, freilich immer wieder modifizierten Grundgedanken der während der letzten Jahrzehnte praktizierten Wettbewerbspolitik zugrunde. ${ }^{10}$

Typisch dafür sind Sätze wie der folgende, der sich auch heute noch in gängigen Lehrbüchern der Volkswirtschaftslehre findet: „Ein ganz wesentlicher Bereich, in dem der Markt versagt, sind die Monopole und andere Formen des unvollständigen Wettbewerbs" (Samuelson und Nordhaus 1998, S. 55). Bei der Diagnostizierung eines solchen angeblichen Marktversagens wird in der Regel zunächst überhaupt nicht unterschieden, ob es sich um Staatsmonopole handelte oder um solche, die auf eigener wirtschaftlicher Leistung beruhen. Das ist ein wesentlicher Unterschied, der aber in der Behandlung der Monopole und ihrer angeblichen Schädlichkeit oft gar nicht zur Sprache kommt - auf der Grundlage des Modells vollständiger Konkurrenz ist er in der Tat auch unerheblich (was bereits ein entscheidendes Argument gegen das Modell ist). ${ }^{11}$ Bei Erhard fällt auf, dass sein Wettbewerbskonzept einzig und allein die angeblich konsumentenschädigende allokative Wirkung von höheren Erträgen und Renten aufgrund von Kartellund Monopolbildungen berücksichtigt, nicht aber die wohlfahrtssteigernden Effekte möglicher Innovationsursachen und -folgen solcher Renten. Themen wie technologischer Fortschritt und Innovation, die sehr viel mit der Entstehung von (effizienten und wohlstandssteigernden) Monopolen und monopolistischem Wettbewerb zu tun haben, kommen bei Erhard nicht vor, stehen jedenfalls

9 Vgl. Pigou (1932/2013).

10 Mantzavinos 2006, S. 284 ff. für eine kritische Darstellung der verschiedenen Varianten des Modells (klassische Preistheorie, der industrieökonomische Ansatz und die spieltheoretische Neuformulierung); für eine kurze Darstellung und Kritik der neoklassischen Grundlagen der EU-Wettbewerbspolitik: Stamate-Ștefan und Mihai-Vladimir 2015.

11 Man muss allerdings einräumen, dass Eucken den Unterschied zuweilen nennt, z.B. Eucken (1952), S. 292 oder S. 334 (wo kurioserweise eigentlich nur die staatliche Privilegierung von Monopolen als das Problem bezeichnet wird). 
nicht im Vordergrund. Darin liegt, wie wir sehen werden, die Achillesferse der Erhard'schen Konzeption.

\section{Die Freiburger Schule und das Modell der vollständigen Konkurrenz}

In seiner auf der Grundlage des neoklassischen Gleichgewichtsmodells der vollständigen Konkurrenz ruhenden Argumentation, Monopole und Kartelle seien die eigentlichen Feinde des Wettbewerbs und damit des Konsumenten, stand Erhard im Einklang mit der von Eucken begründeten Freiburger Schule des Ordoliberalismus. Erhard sprach von vollständiger Konkurrenz als von einem Denkmodell, aufgrund dessen wettbewerbs- und damit wohlfahrtseinschränkende Marktstrukturen und unternehmerische Praktiken erkannt und entsprechend verboten werden könnten (Erhard 1964, S. 176). Das Modell diente inm als eigentliche theoretische Begründung, einer ganz bestimmten Auffassung von sozialer Marktwirtschaft, die sich vom Mainstream der Wohlfahrtsökonomie in der Tradition von Pigou und später Paul A. Samuelson abhebt: Nicht Korrektur von Marktversagen durch staatliche, eventuell auch umverteilende Eingriffe, war Erhards Konzept, sondern Sicherstellung eines nicht durch Kartelle und Monopole verzerrten und damit eben sozialen, d.h. allen Konsumenten zugutekommenden Wettbewerbs.

Was genau ist vollständige Konkurrenz? Sie beschreibt einen Zustand des Gleichgewichts, in dem kein Produzent stärker, besser, innovativer, klüger, wissender, größer, mächtiger als seine Konkurrenten ist und deshalb auch kein Unternehmer bestimmenden Einfluss auf das Marktgeschehen - Marktmacht - insbesondere die Preisbildung haben kann; ein Zustand zudem, in dem alle Produkte homogen sind, es also keine Produktdifferenzierung gibt (z.B. nur einen Typ von Auto). Es ist ein Zustand vollkommenen Gleichgewichts - deshalb aber notwendigerweise auch des technologischen Stillstands, ohne Innovation und evolutionärer Dynamik. Sobald ein oder mehrere innovative Unternehmer alle anderen hinter sich lassen, besteht keine vollständige Konkurrenz mehr, also auch keine Gleichheit der Stimmengewichte. Das wirtschaftliche Gleichgewicht ist auf Kosten des Konsumenten gestört. Mit anderen Worten: Das Modell der vollständigen Konkurrenz beschreibt einen Gleichgewichtszustand im „Jetzt“, ohne Berücksichtigung, was vorher war oder nachher sein wird, atemporal und statisch. Dabei blendet es notwendigerweise aus, was zum Wesen von wirtschaftlichem Wachstum und Wohlstandssteigerung gehört: das unternehmerische Handeln und damit das eigentliche Subjekt des
Wettbewerbs. Das sind natürlich äußerst ungünstige Modell-Voraussetzungen, um überhaupt verstehen zu können, was unternehmerischer Wettbewerb seinem Wesen nach ist, und daraus Grundsätze für die Wettbewerbspolitik zu entwickeln.

Eucken charakterisiert vollständige Konkurrenz ${ }^{12}$ u.a. als einen Zustand, in dem es keine Sperren gibt, weil keine Oligopolisten oder Monopolisten andere daran hindern, am Wettbewerb teilzunehmen. In der vollständigen Konkurrenz gebe es keinen Kampf Mann gegen Mann. Vielmehr vollzieht sie sich „in paralleler Richtung. Sie ist nicht Behinderungs- oder Schädigungswettbewerb, sondern ,Leistungswettbewerb“" (Ebd.) ${ }^{13}$. Erneut findet sich hier das Problem, dass das Modell quer zur Wirklichkeit liegt: Es gehört zum Wesen von Innovation und kreativer unternehmerischer Effizienz, dass sie de facto oft Sperren errichten, also wegen der Neuheit und Einzigartigkeit von Produkten, Produktionsverfahren oder Dienstleistungen den Marktzugang für Konkurrenten erschweren, ja für eine gewisse Zeit ganz verhindern können. Das ist kein Schädigungswettbewerb, sondern die Folge echter Innovation und dadurch unweigerlich erlangter Marktmacht. Natürlich gibt es auch eindeutigen Schädigungs- oder Behinderungswettbewerb, etwa die Errichtung physischer Sperren für den Zugang zu nicht im rechtmäßigen Eigentum stehenden Ressourcen, oder Schädigung der Konkurrenz durch Verleumdung, Lüge oder Betrug. Solchen Praktiken ist jedoch ohnehin gesetzlich vorgebeugt, weil sie an sich schon strafrechtlich relevant sind. ${ }^{14}$ Doch gerade innovative Leistung und entsprechende Vorteile können die Konkurrenz schädigen und behindern. Innovation verschafft einen Vorsprung und Marktmacht - und damit Ungleichheit der Startchancen. ${ }^{15}$ Eucken wie auch sein

12 Der Erklärung des Modells der vollständigen Konkurrenz und ihrer normativen Bedeutung widmet Eucken viele Seiten seiner Grundsätze; vgl. z.B. Eucken (1952), S. 247 ff., 295 ff.

13 Zu den Ursprüngen der Unterscheidung von Leistungs- und Behinderungswettbewerb s. Böhm (1933/2010), S. 179 ff.

$14 \mathrm{Vgl}$. hingegen die weiter unten diskutierten Vorschläge Hayeks gegen Behinderungswettbewerb sowie Erich Hoppmann, der Wettbewerbsfreiheit als „Abwesenheit von Zwang, Betrug und Irreführung“ (Hoppmann 1972, S. 13) kennzeichnet. In diesem Zusammenhang wäre auch über die Frage zu diskutieren, ob nicht das Patentrecht Sperren errichtet, die zu gesetzlich geschützten und wettbewerbsverzerrenden Monopolen führt, insbesondere Sperrpatente (Eucken 1952, S. 41, 268 f.). Eine detailliert argumentierende Kritik von Patenten als intellectual monopoly findet sich bei Boldrin und Levine (2008).

15 Überblickt man etwas Beispiele von Behinderungswettbewerb an Fallbeispielen des Bundeskartellamts, beschleicht einen das Gefühl, hier maße sich eine Behörde ein Wissen an, das sie nicht besitzen kann, etwa in der Beurteilung von Treuerabatten als reine Behinderungsstrategie ohne Leistungskomponente (vgl. den Fall EffemTierfertignahrung aus dem Jahre 1980, beschrieben bei Schmidt und Haucap 2013, S. 317 ff.). 
wichtigster Mitstreiter Böhm erblickten hingegen in einer Welt von gleichberechtigten und deshalb möglichst kleinen Unternehmen das Ideal einer Unternehmerwelt, in der alle gleiche Startchancen haben. Es ist jedoch keineswegs einleuchtend, weshalb eine echte Wettbewerbsordnung sich dadurch auszeichnen sollte, dass sie ein „Wettkampf mit gleichen Startbedingungen“ (Röpke 1950, S. 21) sein soll. Diese Idee mutet geradezu egalitaristisch an. Natürlich muss Rechtsgleichheit herrschen. Alle müssen den gleichen Spielregeln unterworfen sein. Das ist die liberale Lösung. Hingegen Gleichheit der ökonomischen Startbedingungen zu fordern, entstammt dem Denken in Kategorien der vollständigen Konkurrenz als Ideal und scheint mit einer freien und innovativen Wirtschaft unvereinbar zu sein.

Auf der Ebene des Wettbewerbs oder der Produzenten bedarf es nicht einer der demokratischen Gleichheit analogen Gleichheit der Chancen. Dazu Ludwig von Mises bereits im Jahr 1929: „Es ist Begriffsverwirrung, das Postulat der ,Gleichheit der Bedingungen" aus den Spielregeln des Sports oder aus der Anordnung wissenschaftlicher und technologischer Laboratoriumsversuche auf die Wirtschaftspolitik zu übertragen" (Mises 1929/1976, S. 45). Die Analogie zur politischen Gleichheit gilt nur für die Konsumentenebene. Dort hat jeder Bürger eine Stimme und diese entscheidet - in der Regel zugunsten der Innovativsten, Effizientesten und deshalb oft auch Größten. Marktmacht wird vom Konsumenten verliehen und ist dann die Belohnung für unternehmerische Leistung.

Eine Welt der staatlich überwachten vollständigen Konkurrenz wäre eine Welt, in der die Schwächeren, das heißt die weniger Effizienten, vor den Stärkeren, das heißt den Besseren, Effizientesten, Innovativsten, durch den Staat geschützt werden. Diesen „Zustand allseitiger Machtlosigkeit in idealer Vollkommenheit“ (Böhm 1947/2007, S. 69) aller Wirtschaftspolitik als Leitprinzip zugrunde zu legen, war für die Ordoliberalen Programm. Eucken formulierte zwar etwas vorsichtiger als Böhm, aber im Wesentlichen gleich: Es sei eine positive Wirtschaftsverfassungspolitik notwendig, „die darauf abzielt, die Marktform der vollständigen Konkurrenz zur Entwicklung zu bringen und so das Grundprinzip zu erfüllen“ (Eucken 1952, S. 255). Dazu seien Konzerne „soweit wie möglich aufzulösen" (Eucken 1947/2012, S. 79) und die Behörde sollte „die Strategie verfolgen, alle wirtschaftlichen Machtgebilde dahingehend zu beeinflussen, sich so zu verhalten, wie sie dies unter Bedingungen eines vollständigen Wettbewerbs tun würden" (Ebd.).

Hans Otto Lenel argumentierte, Eucken habe lediglich eine wettbewerbspolitische Konzeption des als ob vertreten (Lenel 1975). Wie aus manchen Texten hervorgeht, war sich Eucken in der Tat bewusst, dass ein Zustand vollständiger Konkurrenz in der Wirklichkeit nie erreichbar sein wird. Dennoch erscheint seine oben erwähnte Forderung nach einer positiven Wirtschaftsverfassungspolitik, die „darauf abzielt, die Marktform der vollständigen Konkurrenz zur Entwicklung zu bringen und so das Grundprinzip zu erfüllen“ (Eucken 1952, S. 255), mehr als eine Wettbewerbspolitik des bloßen als ob. Positive Wirtschaftsverfassungspolitik heiße nämlich - so Eucken an gleicher Stelle -, dass es nicht genüge, „dass gewisse wirtschaftspolitische Akte vermieden werden: so etwa staatliche Subventionen, Herstellung staatlicher Zwangsmonopole, allgemeiner Preisstopp, Einfuhrverbote usw. Es genügt auch nicht Kartelle zu verbieten. Das Prinzip ist nicht in erster Linie negativ“ (Ebd.). ${ }^{16}$

Nun war Eucken gewiss kein Doktrinär. Er wusste zwischen Prinzip und Moment, der in jeder historischen Situation angemessenen Verwirklichung des Prinzips jenseits von Doktrinarismus und ungrundsätzlichem Punktualismus zu unterscheiden (Eucken 1952, S. 251). Doch mit Böhm (und Rüstow, Röpke u.a.) erblickte er das zu erstrebende Ideal in einer Welt kleiner Unternehmen, die in vollständiger Konkurrenz stehend sich um die Gunst der Konsumenten bemühen. Große Konzerne und monopolistische Konkurrenz erschienen in einem solchen Licht als Störfaktoren für Wettbewerb, Wohlstand und Freiheit. Zudem trat Eucken für eine Welt ein, in der Unternehmer immer auch Eigentümer ihrer Unternehmen sind. Ein Unternehmertum, das von fremden Kapitalgebern abhängig und entsprechend in seinen Entscheidungen nicht unabhängig wäre, lehnte Eucken als Widerspruch mit den Idealen einer echten Marktwirtschaft, eines selbstbestimmten, verantwortlichen Unternehmertums und des fairen Wettbewerbs $a b$ - eine Idee, die heute neokommunistische Vertreter einer Marktwirtschaft ohne Kapitalismus wie Sahra Wagenknecht aufgreifen, um, mit Berufung auf Adam Smith und Eucken, Kapitalgesellschaften und das Modell beschränkter Haftung zu diskreditieren (Wagenknecht 2016, S. 257).

16 Vgl. auch Eucken (1952), S. 292 f.: es geht nicht nur um Kartellverbote, sondern um eine „Wirtschafts- und Rechtspolitik“ (Ebd.), die die „konstituierenden Prinzipien zum Durchbruch bringen“ (Ebd.). Dadurch befreie sich der Staat weitgehend vom Einfluss privater Machtgruppen - in der Tat ein wichtiges Anliegen. Die Frage ist nur, ob das einzige Mittel, um Politik und Gesetzgebung von solchem Einfluss frei zu halten, in der Zerstörung bzw. Verhinderung der Entstehung marktmächtiger Unternehmen besteht. Dazu fehlt zumindest ein Glied in der Begründungskette - es sei denn, man gehe vom Idealbild der vollständigen Konkurrenz aus, wodurch die Begründung, nämlich die prinzipielle Schädlichkeit von Marktmacht, bereits durch das Modell vorgegeben wird. 


\section{Erhard und die Ordoliberalen: Übereinstimmungen und Differenzen}

Gemäß Horst Wünsche betrachtete Erhard die Vorstellung einer Wettbewerbsordnung, die aus dem bloßen Modell eine zu verfolgende Politik macht, als „akademisch und weltfremd" (Wünsche 2015, S. 68). Er habe „niemals erwogen, seine Politik an ihr zu orientieren“ (Ebd.). Diese Aussage scheint insofern richtig, als Erhard aus dem Modell der vollständigen Konkurrenz nicht wie Eucken einen strategischen Punkt machte. Wünsches Interpretation kann aber entgegengehalten werden, dass Erhard seine Politik eben doch an der Vorstellung vollständiger Konkurrenz orientierte. Das Modell lieferte inm die Richtschnur für seine Antikartell- und Antimonopolpolitik und auch er forderte vom Gesetzgeber, dass er "die vollständige Konkurrenz in einem möglichst großen Umfang erhält" (Erhard 1964, S. 173). Insofern muss der Versuch Wünsches, Erhard in Distanz zur Neoklassik und ihrem Modell der vollständigen Konkurrenz zu rücken, ja zu behaupten, er habe gegenüber den Ordoliberalen eine andere, eigenständige wissenschaftliche Konzeption vertreten, unhaltbar erscheinen. ${ }^{17}$ Die wettbewerbstheoretische Grundlage von Erhards Wettbewerbs- und Wohlfahrtskonzeption gehörte eindeutig dem neoklassischen Mainstream an. ${ }^{18}$

Besser als mit einer eigenen wissenschaftlichen Konzeption, lassen sich die Differenzen von Erhards Auffassung zu derjenigen der Freiburger Schule dadurch erklären, dass er, Erhard, gerade keine geschlossene wissenschaftliche Konzeption hatte, sondern, zwar befruchtet vom Ordnungsdenken der Freiburger Schule, eine vorwiegend politisch umsetzbare Konzeption entwickelte. Sie speist sich aus mehreren, darunter nicht nur wissenschaftlichen Quellen, enthält sicher auch pragmatische Elemente, stimmte aber mit der von den Freiburgern vertretenen Konzeption im Eintreten für eine freie und auf Selbstverantwortung basierende Wirtschafts- und Gesellschaftsordnung überein.

So kann es nicht erstaunen, dass Erhard sich wiederholt auf die Freiburger Schule als seine Verbündeten und Gleichgesinnten berief. Sie vertraten das neoklassische Denkmodell der vollständigen Konkurrenz, das ja, wie erwähnt, Erhards früherer Lehrer Wilhelm Rieger abgelehnt hatte. Freie oder unbeschränkte

17 So auch Dörr (2015).

18 Was insofern erstaunt, als Erhards Lehrer Wilhelm Rieger die neoklassische Gleichgewichtstheorie abgelehnt hatte (Wünsche 2015, S. 290). Das ist aber noch kein Argument dafür, dass dies bei Erhard ebenfalls der Fall war, zumal, wie wir gleich sehen werden, hier Oppenheimer durchaus erstaunlich neoklassisch dachte.
Konkurrenz spielte jedoch in Oppenheimers Denken eine zentrale Rolle: sie war für inn das ökonomische Prinzip der "Selbststeuerung des Marktes" (Oppenheimer 1938/1998, S. 468), so dass, wo „keinerlei Monopolverhältnisse einspielen [...] die freie Konkurrenz die Einkommen auf die Dauer und im Durchschnitt so weit ausgleichen, wie das der relativen Qualifikation der Arbeitskraft entspricht" (Ebd., S. 471, Hervorhebung im Original). Das klingt nach Erhard avant la lettre: Wohlstand für alle durch Wettbewerb. ${ }^{19} \mathrm{Im}$ Jahr 1971, in der Festschrift zum 90. Geburtstag von Mises, betonte Erhard, dass es die Freiburger Schule des Ordoliberalismus, insbesondere Eucken, war, die dem Kernübel des klassischen Liberalismus entgegengetreten war, nämlich „der mangelnden Berücksichtigung der seinerzeit wohl gültigen, aber auf die Dauer immer unhaltbarer werdenden gesellschafts- und rechtspolitischen Auffassungen" (Erhard 1971/1988, S. 1042), was eine „schonungslose Ausnutzung der menschlichen Arbeitskraft ermöglichte" (Ebd.). Der Ordoliberalismus der Freiburger Schule habe nicht nur "das Wettbewerbsbewusstsein neu belebt" (Ebd., S. 1043), sondern Dank ihrer Erkenntnisse habe auch "das Ordnungselement des Wettbewerbs [...] wieder einen besten Standort innerhalb der freien Marktwirtschaft zugewiesen bekommen“ (Ebd.). ${ }^{20}$ Diese Äußerung kann als Bestätigung für die Ansicht von Patricia Commun gelten, Erhard habe „erst mit Hilfe der Ordoliberalen“" (Commun 2015, S. 313) die Bestandteile seiner bisherigen Erfahrungen "zu einer kohärenten Ordnungspolitik“ (Ebd.) zusammensetzen können. Das mag richtig sein, was Erhards Vorstellungen einer Antikartell- und Antimonopolgesetzgebung betrifft, nicht aber, was sein Vertrauen in die freien Marktkräfte anbelangt, das - wohl unter Einfluss Oppenheimers - im Vergleich mit den Ordoliberalen und ihrer Kritik an Laissez faire ungleich höher war.

Auch in seiner Gedenkrede zum 100. Geburtstag seines Lehrers Franz Oppenheimer im Jahr 1964,

19 Haselbach (1985), S. 158 sieht zwischen Oppenheimers Begriff der freien Konkurrenz, die sich in Abwesenheit von Monopolen von allein einstellt, und der "vollständigen Konkurrenz" der Ordoliberalen, deren Wahrung des staatlichen Eingriffs bedarf, einen krassen Gegensatz, was in der Tat insofern zutreffend ist, als für Oppenheimer der Zustand des freien Wettbewerbs (oder unbeschränkter Konkurrenz) keine Folge einer staatlichen Wettbewerbspolitik, sondern eher Folge des Verzichts auf Politik ist (da ja für inn Monopole letztlich auf der Politik der Bodensperre beruhen, eine problematische Lehre, die an dieser Stelle nicht diskutiert werden kann).

20 Bemerkenswert ist, dass Erhard hier von freier Marktwirtschaft spricht (Ebd., S. 1050), wo er vor einer „falschen Ausdeutung des Begriffs ,sozial““ (Ebd.) warnt; Folge sei „die Gefahr, durch einen immer stärker werdenden Dirigismus bzw. einen immer mächtiger aufkommenden Kollektivismus nunmehr von Staats wegen manipuliert zu werden." (Ebd.). 
verwies Erhard auf die Freiburger Schule als seine geistigen Verbündeten:

„Man reiht mich gemeiniglich ein in die Kategorie der ,Neoliberalen'. Es mag so geschehen; ich wehre mich gar nicht dagegen, denn Gelehrte von Walter Eucken angefangen über Wilhelm Röpke, Alexander Rüstow zu Friedrich August von Hayek und Böhm, um nur einige zu nennen, haben im tiefsten Grund Oppenheimersches Gedankengut in sich aufgenommen und in unsere Gegenwart übersetzt, in dem sie einen leidenschaftlichen Kampf gegen die Beschränkungen des Wettbewerbs und vor allen Dingen gegen Monopole führten“ (Erhard 1964/1988, S. 861).

Dieses Lob der Freiburger Schule klingt allerdings etwas distanziert (,ich wehre mich nicht dagegen“). Zudem unterstreicht es nicht, was er, Erhard, den Ordoliberalen verdankt, sondern umgekehrt, was die Ordoliberalen Oppenheimer verdanken! Wie weit solche Äußerungen an Festakten Aufschluss über reale wirkungsgeschichtliche Zusammenhänge geben können, bleibe dahingestellt. ${ }^{21}$

Eines erscheint jedoch klar: Das den Ordoliberalen und dem Schüler Oppenheimers gemeinsame Thema wird im obigen Zitat klar benannt: der Kampf gegen Wettbewerbsbeschränkungen und Monopole. Erhard erblickte in der Marktmacht großer Konzerne, Monopole oder Kartelle die Gefahr von Konsumentenschädigung und Wohlfahrtseinbußen. Missbrauch von Marktmacht, ja Marktmacht selbst müsse deshalb, so Erhard, durch eine staatliche Antikartell- und Antimonopolpolitik, also durch Wettbewerbspolitik mit Hilfe von Kartellbehörden verhindert werden. Auch wenn er das schon von Oppenheimer gelernt hatte, ließ es sich in der Nachkriegszeit, in der sein früherer Lehrer keine Rolle mehr spielte, besser unter Berufung auf die Vertreter der Freiburger Schule legitimieren. ${ }^{22}$

21 Das gilt auch für Erhards Äußerungen beim CDU-Empfang zur Feier seines eigenen 80. Geburtstag, wo er vor versammeltem Festpublikum (darunter die Witwen Euckens und Röpkes, sowie Böhm, Müller-Armack und Hayek) bemerkte, ,ich käme mir geradezu verlogen vor, wenn ich behaupten wollte, dass die geistige Grundlage der Sozialen Marktwirtschaft allein meinem Kopf entsprang" (Erhard 1977, S. 297) und dann die Genannten einzeln erwähnte, und darauf fortfuhr: „wir alle, die uns zum Neo- bzw. Ordoliberalismus bekennen [...]" (Ebd., S. 299).

22 Ebenso nahe stand er Röpke, der Monopole generell mit Staatsbetrieben, Bürokratismus und Kommandowirtschaft gleichsetzte (Röpke 1950, S. 27) sowie behauptete, dass „Monopolbildungen aller Art und jeden Grades den Leistungswettbewerb eindämmen" (Ebd., S. 94). Erhard teilte aber nicht Röpkes fast sozialromantische Ablehnung von Großbetrieben (Ebd., S. 26).
Eine bezeichnende Differenz zu den Freiburger Ordoliberalen - und hierin ist Wünsches Darstellung sicherlich zutreffend - zeigt sich jedoch darin, dass Erhard Euckens Ablehnung der Abhängigkeit von Unternehmern von Krediten und fremdem Kapital nicht mitvollzog, diese vielmehr als „eine alltägliche, unumgängliche Einschränkung der persönlichen Freiheit" (Wünsche 2015, S. 70) des Unternehmers akzeptierte (Ebd.). Davon zu abstrahieren, so Wünsche, erkläre sich dadurch, dass Eucken „nur theoretisch die Ordnung beschreiben will, in der wirtschaftliche Macht keine Rolle spielt und jede wirtschaftliche Entscheidung in Unabhängigkeit von anderen getroffen wird und niemandem darüber Rechenschaft zu geben ist" (Ebd.). Doch dies ist nicht die reale Welt.

Ob Eucken wirklich nur theoretisch eine Ordnung beschreiben wollte, ist freilich zu bezweifeln. Dafür waren seine „Grundsätze der Wirtschaftspolitik“, aber auch andere seiner Texte, wie etwa die Vorträge an der London School of Economics im Jahre 1950 allzu praxisbezogen. ${ }^{23}$ Eucken verstand seine Grundsätze ganz bestimmt als ein praktisch und politisch zu realisierendes Konzept. Als solches war es auch nicht doktrinär oder unflexibel. Gerade deshalb, aber auch nur deshalb, konnten Erhard und die Ordoliberalen im Kampf um die Durchsetzung einer in der Nachkriegszeit von der Bevölkerung breit akzeptierten marktwirtschaftlichen Ordnung natürliche Verbündete sein. So erstaunt auch nicht, dass Erhard von den Freiburgern und dem ihnen nahestehenden Röpke entscheidende Schützenhilfe erhielt ${ }^{24}$ und Differenzen theoretischer Art aber auch hinsichtlich der praktisch-politischen Umsetzung in den Hintergrund treten konnten. Das heißt aber nicht, dass es sie nicht gab.

Erhard war jedenfalls klar, dass das Ideal einer kleinunternehmerischen Welt, wie es Euckens positive Wirtschaftsverfassungspolitik forderte und wie sie auch Böhm, Röpke und noch mehr Rüstow als Ideal vertraten, unrealistisch und letztlich auch unökonomisch war. Erhard erkannte an, dass es in einer innovativen und dynamischen Welt großer Unternehmen und Konzerne bedurfte, nicht zuletzt um der internationalen Wettbewerbsfähigkeit Deutschlands willen, aber auch allein schon deshalb, weil, der, der innovativ und deshalb erfolgreich

23 Sie finden sich unter dem Titel „Vorträge zur „Wirtschaftspolitik“ wiederabgedruckt durch das Walter-Eucken-Archiv (2012), S. 9-77.

24 So in seinem Gutachten „Ist die deutsche Wirtschaftspolitik richtig?“, das dieser $1950 \mathrm{im}$ Auftrag Adenauers für die Bundesregierung verfasste und große Resonanz in der Öffentlichkeit fand; s. Röpke (1950). Es geht aber wenig auf die konkrete Politik Erhards ein, sondern liest sich wie eine davon unabhängige, an eine breitere Öffentlichkeit gerichtete Verteidigungsschrift für eine konsequent markwirtschaftlich orientierte Politik - ohne Zweifel eine Rückendeckung für Erhard und den von ihm eingeschlagenen Weg zu einer Zeit, in der sein Erfolg noch nicht feststand. 
ist, groß wird und gerade dadurch - infolge von Skalenerträgen und anderen Rationalisierungsmöglichkeiten - Marktmacht erhält. Das wiederum heißt nicht, dass nicht auch kleine und mittelständische Unternehmen wesentlich sind. Die Großen brauchen die Kleinen, und umgekehrt. Erhard war diese Durchmischung von Großbetrieben, mittelständischen und Kleinbetrieben zu Recht ein zentrales Anliegen, denn nur so bestünde keine Gefahr eines Monopolismus - eine m.E. sehr richtige Ansicht, die aber wiederum mit Erhards Auffassung, Monopole seien an sich schädlich, im Widerspruch steht.

Eine solche Welt kann gerade nicht durch ein Denkmodell wie dem der vollständigen Konkurrenz erfasst werden - ja widerspricht diesem diametral. Dennoch verteidigte es Erhard geradezu heftig und zuweilen in belehrendem Ton: Wer sich „über das ,Denkmodell' des vollkommenen Wettbewerbs glaubt lustig machen zu sollen, verrät damit nur seine eigene geistige Unzulänglichkeit“" (Erhard 1964, S. 176). ${ }^{25}$ Er verteidigte - trotz gelegentlicher anderslautender Formulierungen (z.B. Ebd., S. 174) auch das eigentliche Mantra der Ordoliberalen: nicht nur der Missbrauch von Marktmacht, sondern Marktmacht selbst sei das Übel und müsse mit Verboten verhindert werden (Ebd., S. 177 ff.). Kartelle seien aus Prinzip schädlich, nicht nur ihr Missbrauch (Erhard 1955/1988, S. 432 ff.). Hingegen war die Position der Ordoliberalen hier immer glasklar gewesen. ${ }^{26}$ Deshalb forderten sie auch die Entflechtung von Konzernen, ein Ansinnen dem sich Erhard vehement und mit Erfolg widersetzte. Hätte er wirklich Marktmacht als solche aufheben wollen, hätte er in diesem Punkt den Ordoliberalen und ihrer Idealisierung vollständiger Konkurrenz folgen müssen. ${ }^{27}$

25 S. auch Erhard 1964, S. 861 f.: , [...] wenn und wo nicht ein vollständiger Wettbewerb besteht, wo immer Konkurrenz durch faktische oder rechtliche Maßnahmen unterbunden, unterdrückt oder geschmälert wird, gibt es keine Freiheit - dort gibt es auch keine Gerechtigkeit.“

26 Vgl. Eucken (1952), S. 172 f.; Böhm (1933/2010); Böhm, (1947/2007), S. 59-91, 75.

27 Gemäß der Darstellung des Wirtschaftshistorikers und Journalisten Werner Kurzlechner, hatte Erhard den stark ordoliberal geprägten Entwurf für ein Wettbewerbsgesetz von Paul Josten aus dem Jahre 1947, an dem auch Franz Böhm mitgearbeitet hatte, schubladisiert. Der Entwurf sah „auch eine Entschachtelung allzu mächtig eingeschätzter Konzerne vor" (Kurzlechner 2008, S. 70). Erhard lehnte Entflechtungen ab, was zeige, dass er „der von Walter Eucken in Freiburg begründeten ordoliberalen Schule zwar in mancher Hinsicht nahestand, ohne jedoch ein bedingungsloser Anhänger zu sein. So entschlossen Erhard war, durch eine Auflösung der Kartelle für eine ,politische Entgiftung' zu sorgen, so entschieden lehnte er Eingriffe in die Wirtschaft wie beispielsweise Entflechtungen ab. Seine Wettbewerbspolitik verschrieb sich nicht sozialromantischen Vorstellungen eines mittelständischen Kleinkapitalismus, sondern wünschte eine international konkurrenzfähige Großindustrie." (Ebd.). Deshalb beauftragte Erhard „als Gegengewicht zu Josten und Böhm [...] eigene Beamte wie Roland Risse und Eberhard Günther mit Vorarbeiten für ein Kartellgesetz" (Ebd.).
Als vorläufiges Ergebnis können wir festhalten: Sowohl Erhard als auch die Neoliberalen argumentierten auf der Grundlage der Neoklassik und ihres Gleichgewichtsmodells der vollständigen Konkurrenz. Während jedoch die Ordoliberalen aus dem Idealbild der vollständigen Konkurrenz unmittelbar praktische Konsequenzen für eine positive Wirtschaftsverfassungspolitik ableiteten, benutzte Erhard es nur als Denkmodell um auf seiner Grundlage die Errichtung negativer Schranken gegen wettbewerbsverzerrende Monopole und Kartelle zu begründen. Die Annahme scheint erlaubt, Erhard habe die neoklassische Rede von der vollständigen Konkurrenz zwar übernommen, aber eigentlich Oppenheimers Idee der durch Monopole gestörten reinen Konkurrenz gemeint. ${ }^{28}$ Deshalb finden wir bei Erhard auch keine Theorie einer positiven Wirtschaftsverfassungspolitik, sondern lediglich die Überzeugung, wohlfahrtsvermindernde Monopole und Kartelle seien durch gesetzliche Maßnahmen zu verhindern. Mit anderen Worten: Die ordoliberale Konzeption ist eine glasklare Ordnungstheorie, die gleichsam eine andere Welt zu schaffen beabsichtigt, in der Praxis allerdings flexibel sein kann und lediglich beansprucht so weit wie möglich verwirklicht zu werden. Die Erhard'sche Konzeption hingegen ist theoretisch weniger geschlossen und besteht eigentlich nur darin, in der Welt, wie sie ist, durch gezielte Verbote von Monopolen und Kartellen allokative Ineffizienz zu verhindern. Das sind zwei verschiedene Ansätze. Man versteht allerdings, weshalb sie gerade in der praktischen Umsetzung gut nebeneinanderbestehen und sich ihre Vertreter als Verbündete verstehen konnten.

Im Gegensatz zur extremeren, aber auch geschlosseneren und ein normatives Ideal zeichnenden ordoliberalen Position, besteht die Crux der die politischen Bedingungen der Realität berücksichtigenden Erhard'schen Position, darin, dass sie, ihrer Intention als realistische Konzeption, mit dieser Realität umso offensichtlicher in Widerspruch gerät. In Konflikt gerät sie vor allem mit der Wirklichkeit der auch von Erhard nicht als legitim bestrittenen, ja nicht einmal thematisierten, staatlichen Monopolbetriebe aller Art im Bereich von Verkehr, Transportwesen, Kommunikation, Fernsehen usw. mit einer entsprechend ständig zunehmenden Staatsquote. Solche Staatsmonopole sollte es ja zumindest in der idealen Welt des Ordoliberalismus nicht geben und wo es sie gibt, sind sie durch eine staatliche Behörde zu kontrollieren (allein das staatliche Geldmonopol erscheint dem Ordoliberalismus notwendig und über alle

28 Wie weit der in der Tradition der Historischen Schule (und besonders Adolph Wagners) stehende Oppenheimer selbst durch die Neoklassik beeinflusst war, muss hier dahingestellt bleiben. 
Zweifel erhaben). Man muss die Frage stellen, ob nicht gerade ein weitausufernder öffentlicher Dienst, Staatsmonopole verschiedenster Art und die damit einhergehende hohe Staatsquote, aber auch Regulierungen und andere Markteintrittsbarrieren staatliche Eingriffe in das Wirtschaftsgefüge sind, die in einem viel höheren Maße wettbewerbsverzerrend, ja - man denke an das erst vor kurzem gefallene Konkurrenzverbot der Deutschen Bahn - geradezu wettbewerbsverhindernd sind. Dazu ist an das einflussreiche Lobbying der Interessenverbände mit ihrer Einflussnahme auf Staat und Gesetzgebung zu denken, wie es von Mancur L. Olson (Olson 1965/2004 und 1991) analysiert, aber auch von Goetz Briefs kritisiert wurde (Briefs 1966). Auch Nell-Breuning legte - allerdings in ganz anderer Absicht - auf solche Lücken und Inkonsequenzen von Erhards Konzept der sozialen Marktwirtschaft schonungslos den Finger, indem er darauf hinwies, dass in ihr der Arbeitsmarkt, das Bankwesen, das Versicherungswesen, das Verkehrswesen und eine ganze Reihe von öffentlichen Monopol-Unternehmen vom Prinzip des freien Wettbewerbs ausgeschlossen blieben, dieser also keineswegs ein durchgehendes Gestaltungsprinzip sei (Nell-Breuning 1986/1990, S. 230 f.). In Anbetracht dieses Befunds, darf man fragen, ob die Fixierung auf die Bekämpfung von Monopolen und Kartellen nicht bedeutet, aus der Mücke einen Elefanten zu machen und dabei den Elefanten zu übersehen: den wettbewerbsverzerrenden und -verhindernden Staat.

\section{Erhard und die neoklassische Wettbewerbstheorie: eine unglückliche Liaison}

Die Heftigkeit, mit der Böhm und Eucken und mit innen auch Erhard Monopole und Kartelle als das Übel schlechthin denunzierten, war natürlich nicht einfach theoretisch aus dem Modell der vollständigen Konkurrenz abgeleitet. Verständlich wird sie nur auf dem Hintergrund der Kartellierung der deutschen Wirtschaft, wie sie seit dem letzten Drittel des 19. Jahrhunderts bis zum Ende der Weimarer Republik zu einer beispiellosen Verschränkung von Staat und Wirtschaft, ja geradezu einer Usurpation des Staates und der Politik durch Großkonzerne, wirtschaftliche Interessengruppen und Verbände geführt hatte. Eucken benennt das Problem deutlich, wenn er die deutsche Kartellverordnung von 1923 erwähnt: „Handelspolitik, Gesellschaftsrecht, Steuerpolitik, Kartellrecht und Kartellrechtsprechung und darüber hinaus das ganze Wirtschaftsrecht haben dort und damals die Monopolbildung erleichtert und gefördert. Teilweise bestanden sogar Zwangssyndikate" (Eucken 1952, S. 292).
Auch Erhard war geprägt von dieser Erfahrung sowie der Überzeugung seines Lehrers Oppenheimer von der Gefährlichkeit der Monopole und Kartelle. Diese auszuschalten, war Oppenheimers Grundanliegen gewesen. ${ }^{29}$ Ein Reflex davon findet sich in einem Beitrag Erhards aus dem Jahr 1949, der den Titel „Kartelle im Blickpunkt der Wirtschaftspolitik" trägt. Daraus wird deutlich, dass die Negativfolie, auf deren Hintergrund Erhard Antikartellpolitik begründet, „die Gefahren der alten deutschen Kartellpolitik" (Erhard 1949/1988, S. 219) waren (Ebd.). In jenen unglücklichen 30er Jahren habe es in Deutschland über 2500 Kartelle gegeben, eine Erstarrung, die das Land ,in eine ausweglose Krise mit ihren MillionenArbeitslosen-Heeren verstrickte" (Ebd., S. 221). Dabei und dies wird hier von Erhard nicht erwähnt - handelte es sich allerdings nicht um spontane Absprachen, sondern um eine bewusste, vom Rechtssystem teilweise geschützte und mit dem Argument der höheren wirtschaftlichen Effizienz bzw. der Notwendigkeit des Schutzes von Betrieben, die von Absatznöten geplagt waren, verteidigte Kartellierungen, die Erhard zu Recht eine Art unternehmerischer Planwirtschaft nannte (Ebd.).

Auch in „Wohlstand für alle" wird deutlich, dass Erhard gegen diese frühere Mentalität ankämpfte und nicht gegen alternative wettbewerbstheoretische Konzeptionen, die er, großgeworden im Umkreis der Historischen Schule, offenbar nicht kannte. Sein Feind waren nicht die Verteidiger von Laissez faire oder von Theorien des freien Wettbewerbs, sondern eine traditionelle Kartellierungsideologie, die behauptet, Kartellierung, wirtschaftliche Konzentration und Großkonzerne seien an sich die wirtschaftlich effizienteste Produktionsform und müsse politisch und rechtlich gefördert werden. Dieselbe Ideologie herrschte bereits um die Jahrhundertwende in den USA und setzte einen Konzentrationsprozess in Gang, der ohne aktive politische und gesetzgeberische Intervention nie in dieser Weise zustande gekommen wäre. ${ }^{30}$ Auch die Verflechtung

29 Bernhard Vogt erhebt Oppenheimer deshalb zum eigentlichen Vater der sozialen Marktwirtschaft (Vogt 1999).

30 Dazu sei auf Kolko (1963/1977) verwiesen, der m.E. überzeugend nachweisen kann, dass der korporatistische Konzentrationsprozess in der amerikanischen Wirtschaft nicht das Werk des freien Marktes oder von Laissez faire war, sondern Folge einer Politik und Gesetzgebung der sog. Progressive Era, die im Verbund mit maßgeblichen Wirtschaftsführern, darunter auch Bankiers wie J.P. Morgan, und getrieben von der Idee, wirtschaftliche Konzentration bedeute Stärke und mehr Konkurrenzfähigkeit, diese entsprechend betrieben. Große Konzerne hatten daran ein Interesse und machten mit, weil es sie vor der Konkurrenz der Kleineren schützte, aber auch vor dem Zugriff der Kartellpolitik auf Grundlage des Sherman Acts. Kolko zeigt, dass es mit dem Clayton Act aus dem Jahr 1914 in Wirklichkeit der Wirtschaft gelungen war, die Politik für sich einzuspannen. Er fasst das Ergebnis seines Buches folgendermaßen zusammen: „'progres- 
von Wirtschaft und Politik in der Weimarer Republik war keineswegs eine Folge eines allzu freien und ungeregelten Marktes, ganz im Gegenteil: sie war Folge staatlicher Interventionen und der Ideologie „von der maximalen Effektivität der Konzentrationspolitik“ (Bracher 1971, S. 194) $)^{31}$.

Während jedoch Eucken behauptete, auch die unselige Entwicklung am Ende des 19. und zu Beginn des 20. Jahrhunderts sei letztlich eine Folge von Laissez faire gewesen (Eucken 1952, S. 31 f.) war sie in Wirklichkeit das Ergebnis staatlich betriebener Schutzzollpolitik und einer ganzen Reihe von staatlichen Interventionen und Gesetzesmaßnahmen, die schließlich zu ungebändigtem Lobbyismus und einer Verfilzung von Politik und Wirtschaft führten und in Deutschland nach dem Jahr 1933 der Etablierung der NS-Herrschaft dienlich war. Echte Marktliberale wie Mises - ein Befürworter von Laissez faire - prangerten in den zwanziger Jahren des letzten Jahrhunderts diesen staatlichen Interventionismus unerbittlich an. ${ }^{32}$ Und Hayek hatte im Jahr 1943, während des Zweiten Weltkrieges, in seinem Buch „The Road to Serfdom" die Ideologie des Monopolismus deutlich als die eigentliche Ursache des Übels angeprangert (Hayek 1945/2004, S. 42 ff.) und klargestellt, dass nicht private, sondern staatlich gestützte Monopole das Problem sind (Ebd., S. 171 ff.). Sogar Müller-Armack schrieb im Jahr 1946: „Es wurde von der wissenschaftlichen Forschung nachgewiesen, dass die Hauptursachen für das Versagen der liberalen Marktwirtschaft gar nicht so sehr in ihr selbst liegen, als in einer Verzerrung, der sie durch den von außen kommenden Interventionismus seit dem Ende des vergangenen Jahrhunderts zunehmend unterlag." (Müller-Armack 1946/1990, S. 9). ${ }^{33}$ Dennoch hält sich der auch von

sivism' was a part of a big business effort to attain protection from the unpredictability of too much competition" (Kolko 2012).

31 Ein Überblick über die Kartellierung der deutschen Wirtschaft seit Ende des 19. Jahrhunderts findet sich bei Kurzlechner (2008), S. 28-61.

32 Vgl. Mises (1929/1976) und Mises (1932).

33 Müller-Armack (1946/1990), S. 9. Vgl. auch folgende sehr richtige Bemerkungen Alexander Rüstows aus dem Jahr 1945 über die „subventionistisch-monopolistische Durchsetzung und Zerrüttung der Marktwirtschaft“ im späteren 19. Jahrhundert, ein Verdikt, das allerdings zu seiner eigenen ordoliberalen Position in eklatantem Widerspruch steht: „Nun sahen wir aber, dass die letzte und entscheidende Entartung der Marktwirtschaft direkt und indirekt durch gehäufte subventionistische, protektionistische und monopolfördernde Maßnahme des Staates herbeigeführt worden war, d.h. aber durch einen flagranten Verstoß gegen die Grundmaxime des Liberalismus: Laisser-Faire, Laissez Passer [...]. Die entscheidende und katastrophale Selbstruinierung der kapitalistischen Wirtschaft ist nicht in Ausführung des liberalen Wirtschaftsprogramms, sondern in flagranter Zuwiderhandlung gegen die Grundvorschrift dieses Programms zustande gekommen“ (Rüstow 1945/2001, S. 115 f.). Durch die staatlichen Kartellverordnungen und Kartellgesetzgebung seien ordoliberalen Ökonomen - und an anderen Orten auch von Müller-Armack - kultivierte Topos, die Kartellierung der deutschen Wirtschaft beweise, dass ungezügelter Kapitalismus und Laissez faire zur Katastrophe geführt hätten. Diese Sichtweise war auch diejenige Erhards und sie bleibt bis heute dominant und prägt den Diskurs.

Mises und sein Schüler Hayek beurteilten das 19. Jahrhundert ganz anders. Sie verfielen nicht in die von Ordoliberalen nach dem zweiten Weltkrieg typische Verurteilung eines angeblich verheerenden Laissez faire der Vergangenheit, sondern erblickten das Übel im staatlichen Interventionismus. ${ }^{34}$ Sie hatten auch eine ganz andere Vorstellung von Wettbewerb, den sie als dynamischen und evolutionären Prozess, als Entdeckungsverfahren verstanden (Hayek 1968/2003). Eine Ansicht wie diejenige Erhards: „Jede Monopolstellung birgt in sich die Gefahr der Übervorteilung des Konsumenten und lässt zudem den wirtschaftlichen Fortschritt erstarren" (Erhard 1964, S. 168) hat in der Gedankenwelt der Österreichischen Schule in dieser Form keinen Platz. Erhard differenzierte nicht zwischen rein wirtschaftlichen, durch Innovation und Effizienz entstandene Monopolstellungen und solchen, die durch staatlichen Schutz oder Privilegierung entstehen bzw. sich erhalten. Schließlich gibt es in Erhards Denkmodell der vollständigen Konkurrenz - paradoxerweise und ganz im Gegensatz zu seiner Erfahrung und seinem Weltbild - keinen wettbewerbstheoretischen Ort für den innovativen Unternehmer, dem durch seinen Erfolg von den Konsumenten eine dominierende Marktmacht verliehen wird. Wie gesagt blendet das Denkmodell der vollständigen Konkurrenz diese Art von Unternehmer notwendigerweise aus. Wenn er und mit inm monopolistischer Wettbewerb dann in der realen Welt erscheinen, stören

gerade „die staatlichen Kontrollorgane selbst, ohne deren Willen und Wissen, in psychologisch auf die Dauer kaum vermeidbarer Weise zu Komplizen der Monopolinhaber“ (ebd., S. 26) gemacht worden. Das ist eine beachtenswerte Analyse, zumal Rüstow zu Beginn dieser Schrift sagt: „Die soziale und wirtschaftliche Katastrophe des wirtschaftlichen Liberalismus war im Wesentlichen eine Folge der Absolutheit, mit der er seine Maxime ,Laissez faire, Laissez passer', so viel an ihm lag, durchführte“ (Ebd.). Was stimmt nun?

34 Laissez faire kritisierte Hayek nur in seiner dogmatischen Form, die jeglichen regelsetzenden Eingriff des Rechtssystems ablehnt (Ebd., S. 30, 47 ff.). In dieser Hinsicht war Hayek weit flexibler als Mises, der hier eher dogmatisch argumentierte. Die Kritik von Olson (1982/1991) an der „klassisch-liberalen Laissez-faire-ldeologie ist bei näherem Zusehen nicht gegen eine wirklich freie Marktwirtschaft gerichtet, sondern nur gegen die Ansicht, Laissez faire habe sich als dynamisch stabil erwiesen (gerade auch im Hinblick auf schädliche Kartellbildungen) (Olson 1982/1991, S. 233 ff.). Eine gemäßigte Form von Laissez faire, die dem Staat eine über den Nachtwächterstaat hinausreichende Rolle als regelsetzende Instanz gibt, würde aber wohl mit Olsons Theorie der wohlfahrtsschädlichen Verteilungskoalitionen (wozu er auch Kartelle zählt) kaum in Widerspruch stehen. 
sie das Modell und müssen, damit die Wirklichkeit dem Modell möglichst nahekommt, bekämpft werden.

Genau deshalb kann in einer wohlstandsorientierten Konzeption von Marktwirtschaft und Wettbewerb vollständige Konkurrenz nicht ein zwar unerreichbares, aber "in einem möglichst großen Umfang“ (Erhard 1964, S. 173) zu realisierendes Ideal sein. Es kann weder als Ideal noch als bloßes Denkmodell taugen, weil es ein Zerrbild der Wirklichkeit bietet und den Begriff des wohlstandsschaffenden Wettbewerbs verfälscht. Es kann allein im Sinne Mises' als Denkmodell für den plain state of rest gelten: eine Momentaufnahme des Marktes, bei der ausgeklammert wird, was vorher und was nachher war. Hier hat man es nur mit historischen Preisen (Vergangenheitspreisen) zu tun. Der sich in der Zeit vollziehende Preisbildungsprozess selbst fällt aus dem Raster. Ähnlich auch die von Mises skizzierte Anti-Wirklichkeit der evenly rotating economy: ein Gleichgewichtszustand, der nie bestehen kann, weil unternehmerisches und wirtschaftlich erfolgreiches Handeln sich immer nur aus Zuständen des Ungleichgewichts heraus entwickelt und auf immer wieder neue Ungleichgewichte abzielt, was den evolutionären und innovativen Prozess des wettbewerblichen Entdeckungsverfahrens weitertreibt. Marktwirtschaftliche Preisbildung vollzieht sich innerhalb dieses dynamischen Prozesses und nicht in einem Gleichgewichtszustand. Weil in inm der Faktor Zeit ausgeklammert wird, ist das Gleichgewicht der evenly rotating economy eine "imaginary construction, not a description of reality" (Mises 1949/1998, S. 246). ${ }^{35}$ All das bedeutet, dass Marktprozesse in Gleichgewichtsmodellen nicht wirklich abgebildet werden können, weil diese in der puren Statik verharren und das dynamische und deshalb unvorhersehbare Geschehen verfälschen ${ }^{36}$ Mit anderen Worten: Wettbewerb ist in seiner vollkommensten, nämlich innovativen Form nur im Ungleichgewicht denkbar und kann genau deshalb durch reine Gleichgewichtsmodelle nicht erfasst werden.

35 In der ursprünglichen (mit der späteren amerikanischen nicht ganz identischen) deutschen Ausgabe von "Human Action“ spricht Mises vom „endlichen Ruhezustand und der gleichmäßigen Wirtschaft" (Mises 1940/2010, S. 235 ff.).

36 Ein treffendes Beispiel findet sich in Euckens Londoner Vorträgen (Eucken 1950/2012, S. 21), wo er gegen den angeblichen technologischen Vorteil von Monopolisten durch hohe Investitionen in ihren Maschinenpark als Gegenargument ins Feld führt, dadurch würden anderen Produktionszweigen Produktionsmittel entzogen und die Konsumentenversorgung deshalb verschlechtert. Das stimmt sicher ceteris paribus und in einer statisch-atemporalen Betrachtungsweise - aber nicht unbedingt, wenn Wettbewerb als dynamischer Prozess verstanden und die durch den Monopolisten hervorgebrachte technologische Innovation mitberücksichtigt wird ( $v g l$. dazu unten das Beispiel Standard Oil).
Das diese Differenzierungen ausblendende Denkmodell der vollständigen Konkurrenz zum Beurteilungsmaßstab für die Schädlichkeit von Monopolen und Kartellen zu erheben und staatliche Wettbewerbspolitik danach auszurichten, läuft deshalb letztlich auf den Kampf gegen die Erfolgreichen und Innovativen hinaus. Und dieser Kampf, so lehrt uns die Geschichte, wird oftmals angetrieben durch die weniger erfolgreiche Konkurrenz, die sich dazu, auch unter Zuhilfenahme des Drucks der öffentlichen Meinung, der staatlichen Kontrollorgane und Behörden bedient (so wie heute die EU versucht, Googles Wettbewerbsvorteile zu beschneiden, die aufgrund erfolgreicher und von den Konsumenten honorierter Innovation existieren, um damit die weniger erfolgreiche europäische IT-Industrie zu protegieren). Das nie auszuschließende Profilierungsstreben der Exponenten solcher Behörden bei diesem angeblichen Schutz der Konsumenten trägt schließlich das Ihre dazu bei, innovationsfeindliche Strukturen zu erhalten.

Gewiss: Gerade Erhard sah die Gefahr von Übertreibungen. Deshalb betont er wiederum beschwichtigend, dass sein Entwurf für ein Kartellgesetz frei von Dogmatismus sei und auch gar nicht von der „viel kritisierten Idee der vollständigen Konkurrenz" (Ebd., S. 176 f.) ausgehe (Ebd.). Man sehe „sowohl Konditionenkartelle als auch Exportkartelle vor, und selbst Rationalisierungskartelle dürfen wirksam werden. Niemand kann also guten Gewissens behaupten, dass dem berechtigten Bedürfnis der Wirtschaft nicht Rechnung getragen ist" (Ebd., S. 177). Das war in der Tat eine erhebliche Abweichung vom Ideal der vollständigen Konkurrenz. War es prinzipienloser Pragmatismus oder entsprang diese Inkonsequenz nicht doch Erhards Einsicht, dass die Wirklichkeit der Wirtschaft dem Modell der vollständigen Konkurrenz eben doch nicht gerecht wird? Oder war es der Druck des BDI, des mächtigen Bundesverbandes der Deutschen Industrie und von Adenauer selbst, denen Erhard schließlich nachgeben musste? Das scheint nicht der Fall gewesen zu sein. Zwar bestand dieser Druck, doch hatte Erhard im offensichtlich widerstanden (Commun 2015, S. 315). Warum aber hielt er dann an diesem Denkmodell so verbissen fest und verteidigte es gegen seine Kritiker? Eine mögliche Antwort ist: Er kannte, abgesehen von Oppenheimers Lehre von der reinen Konkurrenz, keine alternativen Denkmodelle. Die Tradition der Österreichischen Schule der Nationalökonomie war inm, der im akademischen Milieu der Historischen Schule großgeworden war, offensichtlich ungenügend bekannt. Man kann das bedauern, denkt doch die Österreichische Schule gerade nicht in weltfremden Gleichgewichtsmodellen, sondern stellt die Analyse des realen unternehmerischen Handelns in den 
Mittelpunkt. Dem Ansatz Erhards hätte dies eigentlich sehr viel mehr entsprochen.

Dass Erhard die Österreichische Schule - zumindest ihr Verständnis von Wettbewerb - nicht wirklich kannte, zeigt ein bezeichnender Lapsus. In seiner bereits oben zitierten Jubiläumsrede zum 100. Geburtstag Oppenheimers ordnete er Hayek dem Ordoliberalismus zu. Sie alle, sagte Erhard über die Neoliberalen ${ }^{37}$, „von Walter Eucken angefangen über Wilhelm Röpke, Alexander Rüstow zu Hayek und Franz Böhm, um nur einige zu nennen" führten - so Erhard - in der Tradition Oppenheimers einen "leidenschaftlichen Kampf gegen die Beschränkungen des Wettbewerbs und vor allen Dingen gegen Monopole" (Erhard 1964/1988, S. 861). ${ }^{38}$ Doch kommt Hayek hier zu falschen Ehren. Ganz bestimmt führte Hayek keinen leidenschaftlichen Kampf gegen Monopole. Die Entscheidung, welche Monopole bzw. monopolistischen Praktiken nützlich und für den Konsumenten ein Gewinn sind, wollte er wie alle Österreicher - weitestgehend dem Markt bzw. den wirtschaftlichen Akteuren überlassen (wir kommen darauf zurück).

Um einem möglichen Missverständnis vorzubeugen, sei erneut betont: hier wird nicht staatlichen oder gesetzlich verliehenen und geschützten Monopolen, die in der Regel tatsächlich schädlich sind, das Wort geredet. Die Rede ist vielmehr von marktbeherrschenden Unternehmen, die von Leuten wie Vanderbilt, Rockefeller, Carnegie oder Ford und heute von Firmen wie Microsoft, Apple, Google oder Amazon geschaffen wurden. Sie errangen sich durch Technologiesprünge und Effizienzgewinne eine Monopolstellung, veränderten dabei aber die Welt und bereicherten die Konsumenten - und hoben auf diese Weise in ungeahntem Ausmaß den allgemeinen Wohlstand an. Auch die heutigen Großen werden, wie es immer geschehen ist, dereinst durch andere, wiederum innovative Konkurrenten abgelöst werden. Auch Monopolisten bzw. marktbeherrschende Firmen sind

37 Erhard erwähnt hier nicht die Freiburger Schule. Er nennt seine Verbündeten meistens die Neoliberalen. Das war gewiss auch eine Selbstbezeichnung der Freiburger (sie stammt von Rüstow), kennzeichnet aber nicht nur die Freiburger Schule, sondern eigentlich alle Mitglieder der aus dem berühmten Walter Lippmann Symposium (1938) hervorgegangenen Mont Pélérin-Gesellschaft, die im Übrigen vor allem durch die Initiative und geistige Mentorschaft Hayeks entstand.

38 In Erhards Rede an der Feier seines 80. Geburtstag, in der er sich auch an „Kollegen von Hayek“ (Erhard 1977, S. 297) richtet, ist die Einordnung Hayeks korrekter aber auch unverbindlicher: „Ich habe Ihre Bücher geradezu verschlungen. Sie haben der Freiheit, die auch ich meine, ein Denkmal gesetzt und sprechen mit mir aus einem Geist“ (Ebd.). Insbesondere erwähnt Erhard hier auch Hayeks Gesinnungsgemeinschaft mit inm in Fragen der internationalen Währungsordnung (Ebd.). dem Wettbewerb ausgesetzt. Entweder werden sie stets besser, verlieren ihre führende Stellung (wie IBM) oder verschwinden (wie Kodak) gänzlich vom Markt.

\section{Vom Nutzen der Monopole: Kritik an der neoklassisch begründeten Wettbewerbspolitik}

Besonders Vertreter der Österreichischen Schule, die mehr im angelsächsischen als im deutschen Raum vertreten sind, gehen mit der klassischen Antikartell- bzw. Wettbewerbspolitik zuweilen hart ins Gericht. Sie wird als policy failure erkannt und ihre theoretischen Grundlagen, die neoklassische Gleichgewichtstheorie und Wohlfahrtsökonomik, als falsch und schädlich kritisiert. So weist beispielsweise einer der führenden Kritiker, Dominick T. Armentano - mit Hayekianischen Argumenten - auf die Widersprüchlichkeit, ja Unsinnigkeit der neoklassischen Theorie der angeblich wohlstandsschmälernden Monopolrente hin (Armentano 1990 und Armentano 2007). Natürlich versucht ein Monopolist, der durch Innovation und Einmaligkeit seines Produkts den Markt dominiert, dieses mit möglichst viel Gewinn zu verkaufen, also nicht zu Grenzkosten oder knapp darüber, wie das bei vollständiger Konkurrenz der Fall wäre. Es ist aber keineswegs einsichtig, dass dadurch wegen überhöhter Preise und verringertem Output eine nur suboptimale Ressourcenallokation und deshalb Wohlfahrtseinbußen entstehen sollen. Ohne diesen innovativen Monopolisten, wäre das Produkt gar nicht oder nur zu einem für die große Mehrheit der Konsumenten unerschwinglich hohen Preis auf dem Markt. Die neoklassische Theorie bezeichnet also paradoxerweise just den Prozess, der Innovation und Wohlstandszuwachs schafft - den eigentlichen Wettbewerbsprozess - als ineffizient und fordert dessen Korrektur durch die Intervention des Staates (Armentano 1990, S. 27).

Das beste historische Beispiel eines wohlstandssteigernden Monopolisten ist John D. Rockefellers Konzern Standard Oil, der wegen seiner innovativen, oft als skrupellos gebrandmarkten und bis heute kontroversen Geschäftsmethoden Opfer einer Verleumdungskampagne und schließlich im Jahr 1911 eines der ersten Kartellverfahren in den USA wurde. ${ }^{39}$ Dieses Verfahren

39 Die klassische Anklageschrift war Ida M. Tarbells reichlich dokumentierte History of the Standard Oil Company, ab 1902 als Artikelserie erschienen, 1904 in Buchform (554 Seiten mit 242 Seiten dokumentarischem Anhang). Von dem Buch existiert eine von David M. Chalmers besorgte kürzere Fassung (Tarbell 1966). Vgl. auch die klassisch-kritische Präsentation Rockefellers als einer der „Robber Barons“ bei Josephson (1934/1995), S. 264-277 ff. Im Folgenden beziehe ich mich auf die Darstellung bei Armentano (1990), S. 55 ff. 
selbst ist legendenumrankt. Der Anklagepunkt war nämlich gar nicht eine angebliche Monopolstellung. Zur Zeit des Verfahrens gab es bereits 147 unabhängige ÖIraffinerien (1908 waren es 125, die Tendenz war also steigend) und Standard Oil besaß nur noch etwa 64\% Marktanteil (1888: 88\%; 1907: 68\%, Tendenz also fallend). ${ }^{40}$ Die Konkurrenz wurde besser, weil sie die technologischen und unternehmerischen Innovationen von Standard Oil zu kopieren begann - ein Lehrbuchbeispiel für den wirtschaftlichen Nutzen von Monopolen.

Standard Oil verbesserte nicht nur innerhalb weniger Jahre in enormem Maße die Qualität von Kerosin, der Konzern vermochte, nach bereits früheren Verbilligungen, zwischen den Jahren 1870 und 1885 den Preis für das stark verbesserte Produkt sogar von 26 auf 8 Cents pro Gallone zu senken (man denke auch an den analogen Fall von Henry Fords Modell T). Diese Effizienzgewinne waren von enormem Nutzen für die Konsumenten, die sich dadurch bedankten, dass sie Standard Oil wie in einem großen Abstimmungsverfahren zeitweise fast $90 \%$ Marktanteil verliehen - nicht, weil Standard Oil die Marktmacht hatte, sondern ganz einfach weil sein Kerosin billiger und besser war und deshalb den Markt beherrschte. Was heißt: Standard Oil wurde von den Konsumenten zum Marktführer gewählt.

$\mathrm{Ab}$ einem bestimmten Punkt ist natürlich die schiere Größe - wegen Skalenerträgen, effizienterer Logistik, Verhandlungsmacht und günstigeren Bedingungen bei Transporttarifen - ein Wettbewerbsvorteil und macht es Konkurrenten für eine gewisse Zeitspanne unmöglich, in das entsprechende Geschäft einzusteigen. Doch tut das nichts zur Sache. Entscheidend ist, dass Standard Oil dem Konsumenten Nutzen und einen beträchtlichen Wohlfahrtsgewinn brachte (ganz abgesehen von der enormen Zahl von Arbeitsplätzen, die dadurch geschaffen wurden, und davon, dass die wegen staatlicher Subventionen stark überbauten und deshalb unrentablen amerikanischen Eisenbahnen ${ }^{41}$ durch die Möglichkeit, Rockefellers Kerosin in alle Ecken der USA zu transportieren, ihre Rentabilität wiedergewinnen bzw. steigern konnten). Deshalb wäre es geradezu unsinnig zu argumentieren, die Tatsache, dass Standard Oil Kerosin zu einem Vielfachen der Grenzkosten verkaufte - mit einer Monopolrente und so viel der Markt eben tragen konnte -, habe zu einer suboptimalen Allokation von Ressourcen oder zu einer Abschöpfung von Konsumentenkaufkraft geführt und bedeute deshalb eine Wohlfahrtseinbuße! Es stimmt zwar, dass Standard Oil zwischen den Jahren 1870 und 1885 die

\footnotetext{
40 Zit. nach Armentano (1990), S. 67, der sich auf Hidy und Hidy (1955) beruft.

41 Vgl. dazu White (2011)
}

Herstellungskosten für (das notabene qualitativ stark verbesserte) Kerosin um das Sechsfache verringern konnte und dass von dieser Einsparung nur etwas mehr als die Hälfte als Preisreduktion an den Konsumenten weitergegeben wurde. Allerdings wurden die dadurch erzielten Monopolgewinne reinvestiert und damit zusätzlicher Konsumentennutzen geschaffen. Es waren Neider und weniger erfolgreiche Konkurrenten, denen es im Verbund mit Politikern wie Theodore Roosevelt in einer beispiellosen Kampagne gelang, die Öffentlichkeit von der Gefährlichkeit dieser Marktmacht zu überzeugen, was schließlich - im Dienste der Gerechtigkeit und demokratischen Gleichheit - zum ersten Antikartellgesetz der USA, dem Sherman Act, führte, aufgrund derer Standard Oil (unterdessen bereits auf nur noch 64\% Marktanteil gesunken!) im Jahr 1911 schließlich zerschlagen wurde.

\section{Dynamische versus statische Betrachtung: Wettbewerb als Prozess schöpferischer Zerstörung und als Entdeckungsverfahren}

Der Fehler der neoklassischen Theorie der angeblich wohlfahrtsverringernden Monopolrente und anderer analoger Vergehen (z.B. Preisdifferenzierung) sowie ihrer falschen Vorstellung von Wettbewerb wird von ihren Kritikern indessen klar benannt: Er beruht darauf, das wirtschaftliche Geschehen als statisches Gleichgewicht anstatt dynamisch, als einen durch die Zeit hindurchlaufenden Prozess zu betrachten (Armentano 1990, S. 22 ff.; Hayek 1946/2003). Natürlich gibt es die Monopolrente. In einer dynamischen Betrachtung des Wettbewerbsprozesses erkennt man jedoch, dass das gesamte Preisniveau durch die auf Innovation und Effizienzsteigerung beruhende Monopolsituation bereits abgesunken ist - zugunsten des Konsumenten. Die durch die Monopolrente erzielten höheren Gewinne dienen dazu, zusätzlich zu investieren, innovativ zu bleiben, durch hohe Dividendenausschüttungen Aktionäre anzuziehen. Solche Monopole durch behördliche Intervention brechen zu wollen, bedeutet in Wirklichkeit, den Konsumenten zu schädigen. Die Ausgaben von Microsoft, Apple, Google oder Amazon im Bereich Forschung und Entwicklung betragen heute bereits ein Vielfaches des gesamten Budgets des EU-Prestigeobjekts CERN kein Wunder, dass solche IT-Giganten staatsgläubigen EU-Bürokraten ein Dorn im Auge sind!

Einer der ersten, der den dynamischen Charakter des Kapitalismus als schöpferische Zerstörung und 
die Nützlichkeit monopolistischer Praktiken sowie die wahre Natur von Wettbewerb klar erkannte, und damit auch den Irrtum des neoklassischen Modells der vollständigen Konkurrenz, war Joseph Schumpeter, ein wie Mises und Hayek aus der Österreichischen Tradition stammender Nationalökonom. Vollkommene Konkurrenz, meinte er, sei „mit der großen Masse dessen, was wir wirtschaftlichen Fortschritt nennen, nicht vereinbar" (Schumpeter 1946, S. 172), ja, sie sei

„nicht allein unmöglich, sondern auch unterlegen und sie kann keinen Anspruch erheben, als Muster idealer Leistungsfähigkeit zu gelten. Es ist deshalb ein Fehler, die Theorie der staatlichen Regulierung der Industrie auf das Prinzip zu gründen, dass die Großunternehmung so arbeiten sollte, wie die entsprechende Industrie es bei vollkommener Konkurrenz täte“ (Ebd., S. 175).

Hayek, der nach seiner Lehrtätigkeit in London und Chicago im Jahr 1962 auf Euckens Freiburger Lehrstuhl zu lehren begann und im Jahr 1974 für seine Konjunkturtheorie den Nobelpreis für Wirtschaft erhielt, verstand, wie bereits erwähnt, den Wettbewerb dynamisch als Entdeckungsverfahren. Im Unterschied zum neoklassischen Gleichgewichtsmodell, in dem der Unternehmer nur als Produktionsfunktion, also gar nicht vorkommt, berücksichtigten diese Ökonomen den Unternehmer und seine Leistung - insbesondere die technologische Innovation - in ihren Wachstums- und Wettbewerbstheorien. Israel M. Kirzner, hervorragender zeitgenössischer Vertreter der Österreichischen Schule in den USA, entwickelte in Abkehr von der neoklassischen Gleichgewichtstheorie eine Preistheorie, die konsequent das unternehmerische Schaffen von neuem Wissen in die Wettbewerbstheorie einbaute und den innovativen Unternehmer wesentlich als Monopolisten sieht (Kirzner 1973, S.22 f.). Hayeks Schüler und Nachfolger auf dem Freiburger Lehrstuhl, Erich Hoppmann, baute die Ansätze seines Vorgängers bis zur Kritik der gängigen Wettbewerbspolitik aus (Hoppmann 1977). Monopolisten oder Quasimonopolisten sind demnach nicht von Übel, vielmehr für den Konsumenten von Nutzen, sofern ihre Marktmacht nicht auf politischer Verfilzung oder gesetzlicher Privilegierung beruht, sondern auf Innovation und Effizienz. Hoppmann hält den Begriff Marktmacht deshalb für unbrauchbar (Ebd., S. 12) und fügt bezüglich Wettbewerbsbeschränkungen hinzu, „dass die gravierendsten Beschränkungen in aller Regel staatlichen Ursprungs sind“ (Ebd. S. 18).

Das neoklassische Gleichgewichtsmodell wurde jedoch während der letzten Jahrzehnte nicht nur von Vertretern der Österreichischen Schule kritisiert und durch ein Denken ersetzt, das der Wirklichkeit einer durch unternehmerisches Handeln und daraus entspringender Innovation strukturierten Wettbewerbs besser entsprach. Alfred Heuss hatte in seiner bahnbrechenden „Allgemeinen Markttheorie“ bereits 1965 auf die "Unhandlichkeit“ des Schumpeterschen, innovativen Unternehmers für die theoretische Analyse hingewiesen, der „einer theoretischen Fixierung schwer zugänglich“ (Heuss 1965, S. 14) und deshalb „von der Marktanalyse weitgehend ausgeklammert worden ist" (Ebd.). Heuss plädierte deshalb für eine differenzierte Markttheorie, die nicht ein uniformes ideales Marktmodell stipuliert, in dem der innovative und deshalb oft monopolistisch agierende Unternehmer, weil er nicht hineinpasst, einfach ausgeklammert wird, sondern die verschiedenen Typen bzw. Phasen von Märkten - die Experimentier-, Expansions-, Ausreifungs- und Stagnationsphase - von den verschiedenen Unternehmertypen her zu verstehen sucht und dadurch auch den Wettbewerb als evolutionären und innovativen Prozess darzustellen vermag (wobei die Ausreifungs- und vor allem die Stagnationsphase wohl am ehesten mit dem Gleichgewichtsmodell der vollständigen Konkurrenz erfassbar ist, der wettbewerbspolitische Versuch - im Sinne des Ordoliberalismus - zur Erhaltung dieses Zustands damit aber auch klar als innovationsfeindlich erkennbar wird). ${ }^{42}$

Auch im angelsächsischen Raum setzte sich im Bereich des wirtschaftswissenschaftlichen Mainstreams vor allem im Gefolge der Arbeiten von Paul Romer eine neue Sicht der Dinge durch, die sogenannte endogene Wachstumstheorie: Neues Wissen und Innovation, die in den neoklassischen Wachstumstheorien als exogen, von außen kommend und damit als wachstumstheoretisch nichterklärbares Residuum behandelt wurden, sind gemäß dieser Wachstumsmodelle selbst Bestandteil des Wachstumsprozesses, nämlich Ergebnis unternehmerischen Handelns und monopolistischen Wettbewerbs im Sinne Schumpeters. Innovatives Wissen ist also eine Ressource, die im unternehmerischen Prozess selbst entsteht (Romer 1986; 1990). ${ }^{43}$ Damit kommt die endogene Wachstumstheorie zu Schlüssen, die das Denken der Österreicher von je her prägten - Information und Wissen sind nicht ein gegebener Input, sondern entstehen gerade erst im Wettbewerbsund Marktprozess - und die für ihre Einsichten in die wohlstandsschaffende Natur von freien Märkten und

42 Vgl. zum Thema auch Mantzavinos (2001), vor allem Kap. 11 und 12; zu Schumpeter und der Schwierigkeit, den Zusammenhangs zwischen monopolistischer Konkurrenz und Innovation empirisch zu bestätigen s. Witt (1987), S. 52 ff.

43 Vgl. auch Jones, Charles I. und Romer, Paul (2009); Grossman und Helpman (1994); Aghion und Howitt (1992); ausführlicher: Aghion und Howitt (2009). Zu Romer auch: Warsh (2006), S 289 ff.; Helpman (2004), S. $36 \mathrm{ff}$. 
unternehmerischem Handeln seit jeher grundlegend waren (Kirzner 1973). Diese Sichtweise wird auch durch die Geschichte bestätigt: Die für Steigerung von Wachstum und Wohlstand entscheidenden technologischen Innovationen haben ihre Quelle nicht außerhalb des unternehmerischen Wettbewerbsprozesses - etwa staatlichen Investitionen in F\&E -, sondern entstammen, wie auch die endogene Wachstumstheorie zeigt, diesem Prozess selbst (Kealey 1996). Natürlich können auch Regierungen, staatliche Forschungsprogramme und Bürokratien Wissenschaft und Forschung organisieren und voranbringen; doch heißt das nicht, dass es nicht auch ohne sie gegangen wäre ${ }^{44}$. Vor allem aber: Wirklich wachstumsfördernder und innovativer technologischer Fortschritt ist endogen, d.h. er stammt aus dem unternehmerischen Wettbewerb selbst. ${ }^{45}$

\section{Folgen der Kartell- und Monopolbekämpfung: Problematischer Machtzuwachs von Staat und Bürokratie}

Erhard gründete, zumindest was seine explizite Argumentation betrifft, seine Konzeption der sozialen Marktwirtschaft als Wohlstand durch Wettbewerb, folglich auf einem Denkmodell, das seinen praktisch-politischen Vorstellungen teilweise widerspricht und mit der auch sein Handeln keineswegs im Einklang steht: die neoklassische Gleichgewichts- und Wettbewerbstheorie. Dennoch hat er diese nie verworfen, sondern, wie er immer wieder betont, für sein Denken als maßgeblich betrachtet. Entsprechend erblickte er die Hauptaufgabe der Wettbewerbspolitik in der Bekämpfung von Kartellen

44 Wie Kealey (1996) zeigt, ist England im 19. Jahrhundert zur führende Wirtschaftsmacht aufgestiegen, obwohl sich das gesamte Bildungssystem in privater Trägerschaft befand; es gab weder staatliche Schulen noch Universitäten und noch weniger staatliche Förderung von Wissenschaft und Forschung.

45 Das ist die Gegenposition zur der - vor allem bei etatistisch denkenden Politikern enorm populär gewordenen - Position von Mazzucato (2014). Zur Kritik an Mazzucato s. Mingardi (2015). Auch wenn staatlich geförderte oder gar getragene F\&E existiert, ist der Staat noch lange nicht unternehmerisch tätig, wie Mazzucato suggeriert. Denn die unternehmerische Tätigkeit besteht nicht darin, Technologien zu schaffen, sondern marktfähige Produkte, die Konsumentenpräferenzen entsprechen und eine Nachfrage generieren können. Hier liegt die eigentliche Innovation. Natürlich war dafür staatlich organisiert F\&E in vielen Fällen nützlich und de facto die Voraussetzung, was aber nicht heißt, dass, wie die Geschichte zeigt, die entsprechenden Technologien nicht ohnehin erfunden und entwickelt worden wären (Ridley 2015, S. 118 ff.). Fragen muss man sich viel mehr, ob steuerfinanzierte F\&E in staatlicher Trägerschaft nicht eher zu enormer Verschwendung von Ressourcen führt. und Monopolen und der Schaffung einer dazu befähigten Kartellbehörde. ${ }^{46}$

Heute stellt sich die Frage, ob letztere - nicht nur in Deutschland - bisher nicht mehr Schaden als Nutzen brachte. Warum soll eine Behörde besser als der Markt, und damit letztlich die Konsumenten, beurteilen können, was den Wohlstand mehrt? Zumal Kartellbehörden immer auf der Ebene politisch eingehegter Wirtschaftsräume (Nationen, EU-Binnenmarkt, TTIP) agieren, gegen außen protektionistisch denken und deshalb das wirkliche Übel nicht anpacken: das Defizit an Freihandel wie auch generell gesetzliche Wettbewerbsbeschränkungen. Auch die Frage nach den Opportunitätskosten einer solchen Politik und der Gefahr des Machtzuwachses von Staat und Bürokratie wäre zu stellen, denn, wie Herbert Giersch im Jahr 1961 gegenüber der ordoliberalen Konzeption bemerkte, korrumpiert Macht und ist eine Bedrohung der Freiheit:

„Insgesamt muss man sich fragen, ob die konsequente Durchführung und erst recht die Durchsetzung der Wettbewerbsordnung im Sinne der ordoliberalen Konzeption nichteineähnlich große Machtfülle des Staates voraussetzt wie eine Zentralverwaltungswirtschaft" (Giersch 1961, S. 185 f.).

Dieses Verdikt impliziert natürlich nicht die Ansicht, die Ordoliberalen hätten tatsächlich eine fast grenzenlose Machtfülle des Staates oder gar eine „Zwangsverwaltungswirtschaft" befürwortet - das genaue Gegenteil ist der Fall. Gierschs Verdikt zielt darauf, auf einen Widerspruch in der ordoliberalen Konzeption aufmerksam zu machen: Die Entmachtung der privaten Wirtschaft ist nur möglich durch einen entsprechenden Machtzuwachs des Staates.

Doch sah auch Eucken das Machtproblem. Deshalb lehnte er eine staatliche Aufsicht über Oligopole ab, weil damit dem Staate zu viel aufgebürdet würde, ein riesiger Kontrollapparat nötig wäre und der Staat „in den alläglichen Wirtschaftsprozess hineingezogen“" (Eucken 1952, S. 298) würde (Ebd.). Auch hinsichtlich der Monopole plädierte Eucken für eine entschiedene Monopolaufsicht, die vor allem „prophylaktisch wirkt“ (Ebd.). Damit fehle Oligopolisten jeglicher Anreiz, „durch Kampf die anderen zu vernichten und eine Monopolstellung zu erobern. Denn dann rennt er in die harte

46 Möglicherweise auch - das wäre zu untersuchen -, weil die alliierten Besatzungstruppen aus politischen Gründen von Anfang an die Auflösung sämtlicher Kartelle und Großkonzerne in Deutschland gefordert hatten, da eben diese Kartellierung der deutschen Wirtschaft es dem Nazi-Regime erleichtert hatte, die gesamte Wirtschaft in eine zentralverwaltete Wirtschaft umzugestalten und damit seine Macht zu festigen. 
Monopolkontrolle hinein“ (Ebd., S. 298 f.). Was aber, so ist dem entgegenzuhalten, wenn ein Unternehmen eine Monopolstellung deshalb erringt, weil es innovativ und besser als die Konkurrenz ist? Auch dann wird er in die Monopolkontrolle hineinrennen - und würde sich deshalb vielleicht davor hüten, allzu innovativ und allzu groß zu werden. Euckens Konzept würde dann also auch auf die Errichtung präventiver Innovationsschranken hinauslaufen.

Auch das wäre allerdings eine Form des - vielleicht verdeckten - Machtzuwachses des Staates und der Wettbewerbsverhinderung, die die prinzipielle Problematik staatlicher Monopol- und Kartellbekämpfung aufzuzeigen geeignet ist: in demselben Maße, wie sie prophylaktisch wirkt, schwächt sie das innovative Potenzial des freien Marktes. Eine staatliche Behörde kann unmöglich beurteilen, ob ein Monopol - dasselbe gilt für Kartelle - schädlich oder nützlich ist. Dazu müsste sie über Informationen verfügen, die nicht gegeben und deshalb niemandem bekannt sind, sondern durch unternehmerische Entscheidungen erst geschaffen werden. Deshalb scheint es effizienter, die Beurteilung ihrer Effizienz dem freien Markt zu überlassen.

Das heißt nicht, dass es ineffiziente und somit für den Konsumenten schädliche Kartelle nicht geben kann oder gibt. Aber in einem offenen Markt ohne Schutzzölle und protektionistische Regulierungen haben konsumentenschädigende Kartelle nicht lang Bestand. Das wusste auch Eucken ${ }^{47}$, es steht klein gedruckt in jedem Lehrbuch und man weiß es natürlich auch aus der Wirtschaftsgeschichte. Olson zitierte das in den USA geflügelte Worte: "Der Zoll ist die Mutter der Trusts“ (Olson 1982/1991, S. 174), denn: „Wenn einmal die Importe ausgeschlossen sind, können die Heimatmärkte auch gewinnbringend kartelliert werden" (Ebd.). Die Kartellierung der deutschen Wirtschaft seit dem Ende des 19. Jahrhunderts war - um erneut daran zu erinnern - durch staatliche Schutzzollpolitik flankiert und erst damit in ihrer Beständigkeit ermöglicht worden. Auch in der heutigen real existierenden Marktwirtschaft sind Schutzzölle und Protektionismus die großen Verbündeten von Kartellen. Auch Erhard erwähnt das - zieht daraus aber keine Konsequenzen für die theoretische Fundierung seiner Politik (Erhard 1964, S. 169). ${ }^{48}$

47 Vgl. Eucken (1952), S. 268.: „In vielen Zweigen der deutschen Industrie werden Kartelle sofort verschwinden, wenn die Zölle fallen.“ Und Ebd. S. 234 (in Bezug auf die Tendenz zu Oligopolen, die zur Kartellbildung neigen): „Mit der Technik verstärkt sich die internationale Konkurrenz auf allen diesen Märkten - wenn sie nicht durch die Wirtschaftspolitik behindert wird" (Hervorhebung nicht im Original).

48 Olson meldet allerdings auch erhebliche Zweifel an, dass Kartelle auch ohne Zölle automatisch verschwinden. Seine historischen Argumente sind aber naturgemäß nicht zwingend (Olson 1982/1991, S. $234 \mathrm{ff}$.)
Kleine, zum Überleben gebildete lokale Kartelle hingegen können durchaus nützlich sein für den Konsumenten und die Produktpalette bereichern; Konsumenten haben ja die Wahl, ob sie beim traditionellen, lokalen und teureren kartellierten Kleinanbieter, oder einem billigeren Großanbieter oder Discounter kaufen möchten. Trotzdem werden die nationalen Kartellbehörden - das Kriterium der sogenannten Unerheblichkeit missachtend - immer mehr gerade auch gegen die Kleineren aktiv, richten dabei vermutlich beträchtlichen wirtschaftlichen Schaden an und verursachen entsprechende Wohlfahrtseinbußen. ${ }^{49}$

Erhards Konzept einer Marktwirtschaft, die sozial ist, weil unternehmerischer Wettbewerb Wohlstand für alle schafft, ist immer noch aktuell und richtig. Korrekturen bedürften aber die wettbewerbstheoretischen Grundlagen eines solchen Konzepts sozialer Marktwirtschaft. Da liegt der Schluss nahe, dass eine Wettbewerbspolitik - abgesehen von der Durchsetzung grundlegender rechtlicher Regeln, welche "die Freiheit des Zugangs zu notwendigen Ressourcen" (Hoppmann, 1977 S. 15) und die „Abwesenheit von Zwang“ (Ebd.) sichern - insofern überflüssig ist, als dass der Markt selbst besser dafür sorgt, dass Wohlstand für alle geschaffen wird. Deshalb kommt der Hayek-Nachfolger Erich Hoppmann zum Schluss: „Die wichtigste Möglichkeit, das Machtproblem zu lösen, wäre das Unterlassen staatlicher Maßnahmen zur Wettbewerbsverhinderung. Für eine spezielle Wettbewerbspolitik gibt es dann nicht mehr viel zu tun." (Ebd.).

Zu dieser Einschätzung ist Hoppmann - wohl unter dem Einfluss Hayeks und in stetem Abrücken von klassisch-ordoliberalen Positionen - allerdings erst allmählich

49 Ein typisches Beispiel ist das Verfahren des Bundeskartellamts gegen verbotene Preisabsprachen bei Bier mit einem Bußgeld in der Höhe von insgesamt 106,5 Mio. $€$ gegen die Unternehmen Bitburger Braugruppe $\mathrm{GmbH}$, Krombacher Brauerei Bernhard Schadeberg $\mathrm{GmbH}$ \& Co. KG, C. \& A. Veltins $\mathrm{GmbH}$ \& Co. KG, Warsteiner Brauerei Haus Cramer KG und Privat-Brauerei Ernst Barre $\mathrm{GmbH}$. „Für Flaschenbier wurde in 2008 eine Preiserhöhung abgesprochen, die zu einer Verteuerung des 20-Flaschen-Kastens von einem Euro führen sollte" (Bundeskartellamt 2014). Das Problem laut einem Pressebericht: „Der deutsche Biermarkt ist seit Jahren rückläufig. 2012 war der Absatz laut Statistischem Bundesamt mit rund 96,5 Millionen Hektolitern auf die niedrigste Menge seit der Wiedervereinigung gesunken. Die Branche liefert sich einen harten Konkurrenzkampf und konnte angesichts der sinkenden Nachfrage in den vergangenen zehn Jahren kaum Preiserhöhungen durchsetzen, während Energiekosten und die Preise für Hopfen und Gerstenmalz deutlich zulegten. Das Bußgeld trifft die Brauer deshalb hart" (n-tv 2014). Man darf sich auch fragen, ob es für Konsumenten nicht ein Vorteil ist, 5 Cents mehr pro Flasche Bier zu bezahlen und dafür eine große Auswahl an lokalen Anbietern zu haben, statt gezwungen zu sein, alle das gleiche, etwas billigere Bier des Großanbieters zu konsumieren (der vielleicht nach Wegfall der Kleinen und damit der lokalen Konkurrenz dann selber die Preise erhöhen wird). Nur der Markt kann entscheiden, was hier besser ist. 
gekommen. ${ }^{50}$ Während er anfänglich (etwa Hoppmann 1968) die eigentliche Gefahr in einem konzentrationsfördernden Laisser faire-Kapitalismus und privater wirtschaftlicher Macht sah und nach einer durchgreifenden Antikartellpolitik und behördlichen Fusionskontrollen rief, lehnte er vier Jahre später im Laufe der sogenannten Hoppmann-Kantzenbach-Kontroverse Fusionskontrollen $a b$, da sie dem freien Ermessen staatlicher Behörden zu viel Macht gäben und - da Wettbewerb ein evolutiver Prozess und ein Entdeckungsverfahren ist - Behörden auch nicht das Wissen dazu besitzen könnten. Vielmehr, so nun Hoppmanns Forderung, seien Fusionen, die voraussichtlich wettbewerbszerstörend sind, generell und präventiv durch entsprechende gesetzliche, sogenannte per se-Regeln zu verbieten. Wiederum später lehnte er auch dies ab: „Der Wunsch, apriorisch ein ex ante lückenloses System von Antimonopolisierungsregeln zu formulieren, ist irrational“ (Hoppmann 1977, S. 29). Dem liegt die Einsicht zugrunde, dass - außer in den Fällen von Betrug, Irreführung und Zwang - die Natur eines dynamisch und evolutorisch verstandenen Wettbewerbs es nicht gestattet, wettbewerbsbehindernde Praktiken von solchen zu unterscheiden, die Ausdruck von Wettbewerbsfreiheit sind.

„Eine Unternehmung ist nicht notwendigerweise wettbewerbsbeschränkend, weil sie groß ist, sondern sie ist möglicherweise groß, weil sie wettbewerblich ist. Ebenso signalisiert auch eine hochkonzentrierte Industrie nicht notwendigerweise das Vorhandensein von Wettbewerbsbeschränkungen, sie kann im Gegenteil hochkonzentriert sein, weil sie wettbewerblich ist" (Ebd., S. 12).

Das zeigt, wie weit sich Hoppmann im Jahr 1977 bereits von seiner Position aus dem Jahr 1968 wegbewegt hatte. Die damals geforderte staatlich veranstaltete Wettbewerbsordnung zur Lösung des angeblichen privatwirtschaftlichen Machtproblems kehrt sich nun um in die Forderung: „Die wichtigste Möglichkeit, das Machtproblem zu lösen, wäre das Unterlassen staatlicher Maßnahmen zur Wettbewerbsverhinderung" (Ebd., S. 18).

Hayek hatte bereits in seinem Buch „Der Weg zur Knechtschaft" die Meinung vertreten, ein Privatmonopol sei „kaum jemals vollständig und noch seltener von langer Lebensdauer oder in der Lage, die Möglichkeit einer Konkurrenz außer Acht zu lassen“ (Hayek 2004, S. 172). Das Gefährliche seien die staatlich geschützten Monopole (Ebd.). Wenn man jedoch ungerechtfertigte Monopolgewinne von Privatmonopolen verhindern wolle, dann besser durch eine Überwachung der Monopole, die auch abschreckend wirkt (hier argumentiert

50 Vgl. zu dieser Entwicklung Eickhof (2009).
Hayek ähnlich wie Eucken). Solche Überwachungen, die man „als Unzulänglichkeiten in Kauf nehmen“ (Ebd., S. 173) könne, „wären kein zu hoher Preis für die Eindämmung von Monopolmacht" (Ebd.) und einer "organisierten Monopolkontrolle" (Ebd.) vorzuziehen.

Im dritten Teil seines Spätwerkes „Recht, Gesetz und Freiheit" (Hayek 2003, S. 391 ff.) finden sich hingegen Vorschläge zu einer regelbasierten Antimonopolgesetzgebung. Vorschläge, die anerkennen, dass Monopole schädlich sein können, nämlich dann, wenn die Fähigkeit „ihre Monopolposition zu schützen und zu erhalten" (Ebd., S. 390 f.) auch dann noch besteht, „wenn die ursprüngliche Ursache ihrer Überlegenheit weggefallen ist" (Ebd.). Sie haben dann die Macht mit inrer Preisgestaltung zu diskriminieren, die Konsumenten zu einem bestimmten Verhalten zu zwingen und Konkurrenten abzuhalten - nicht durch Leistung, sondern durch ihre bloße Machtposition. Doch sei die Verhinderung eines solchen - nicht immer missbräuchlichen und schädlichen - Verhaltens eine schwierige Aufgabe. Es sollte durch entsprechende Verhaltensregeln eingedämmt werden, es sei ,jedoch fraglich, ob sich das wirksam dadurch erreichen ließe, dass man sie zur strafbaren Handlung macht statt lediglich zum Grund für einen Schadenersatzanspruch" (Ebd., S. 391). Eine Behörde besitze nicht diese Art von Wissen, um hier „erfolgreich eine Strafverfolgung zu betreiben“. Deshalb sei es sinnvoller, dass „potentielle Konkurrenten als Aufpasser für den Monopolisten eingesetzt werden und ein Mittel gegen Preisdiskriminierung in die Hand bekommen" (Ebd.), indem sie auf Schadenersatz klagen können, sofern es ihnen gelingt, das zu begründen (Ebd.).

Ähnliches gilt gemäß Hayek auch für Kartelle. Viele seien, als "rein freiwilliger Zusammenschluss von Unternehmen, die nicht mit Zwang operieren, nicht nur nicht schädlich, sondern im Gegenteil vorteilhaft" (Ebd., S. 392). Kartelle dürften aber auch nicht geschützt werden. Anstelle einer behördlichen Missbrauchskontrolle nach Ermessen (sog. rule of reason), wäre es deshalb besser, „ausnahmslos jede Absprache zur Beschränkung des Wettbewerbs für ungültig und rechtlich nicht durchsetzbar zu erklären und alle Versuche, sie durch gezielte Diskriminierung oder dergleichen durchzusetzen, dadurch zu unterbinden, das man, wie oben vorgeschlagen, denjenigen einen mehrfachen Schadenersatzanspruch gewährt, auf die solcher Druck ausgeübt wird“ (Ebd.). ${ }^{51}$ Das Gesetz gegen Wettbewerbsbeschränkungen (GWB) der Bundesrepublik

51 Also genau das Gegenteil dessen, was das deutsche Reichsgericht in seinem folgenreichen Kartellurteil vom Februar 1897 erklärte: Kartellverträge seien grundsätzlich rechtswirksam (außer im Falle von Sittenwidrigkeit, d.h. wenn ihre Absicht die Monopolbildung und Ausbeutung und „wucherische Ausbeutung des Konsumenten“ ist) (Kurzlechner (2008), S. 31; Blaich (1970)). 
Deutschland habe den Fehler begangen es nicht bei der anfänglichen Bestimmung zu belassen, „alle Vereinbarungen zur Wettbewerbsbeschränkung für ungültig erklärt" zu haben, sondern diese mit Strafandrohung zu verbieten, deshalb aber auch gezwungen zu sein, einen ganzen Rattenschwanz von Ausnahmen und Sonderregelungen hinzuzufügen, durch die die allgemeine Regel durchlöchert, der Anwendungsbereich beschränkt und die Durchsetzung behördlichem Ermessen überlassen wird (Hayek 2003, S. 393). ${ }^{52}$ Dass man sich auch für das Gewähren von Ausnahmen aller Art wie auch für deren Nichtgewährung auf die gemäß dem Modell der vollständigen Konkurrenz gefährliche Marktmacht zu berufen pflegt, ist jedem Zeitungsleser bekannt. Weniger im Bewusstsein präsent ist hingegen die damit verbundene Macht der Behörden, aufgrund eines zweifelhaften theoretischen Modells zumindest in vielen Fällen Konsumentennutzen und Wohlfahrtseffekte zu verhindern. ${ }^{53}$

\section{Fazit: Andauernde Aktualität von Erhards Konzept „,Wohlstand durch Wettbewerb“}

Die Welt der vollständigen Konkurrenz ist eine irreale Welt. Das hat Erhard klar erfasst, daraus auch politische Konsequenzen gezogen, ohne die seine Wirtschaftspolitik sicher nicht so erfolgreich gewesen wäre. Aus welchen Gründen auch immer blieb er aber dabei in manchen Punkten dem Denkmodell der vollständigen Konkurrenz und damit der damit verbundenen Idee verhaftet, auch nur faktische, also gesetzlich nicht geschützte Monopole seien - von qualifizierten Ausnahmen abgesehen schädlich und Kartelle müssten in den meisten Fällen durch staatliche Maßnahmen aufgelöst werden.

52 Das GWB von 1998, Fassung 2013, bestimmt in §1: „Vereinbarungen zwischen Unternehmen, Beschlüsse von Unternehmensvereinigungen und aufeinander abgestimmte Verhaltensweisen, die eine Verhinderung, Einschränkung oder Verfälschung des Wettbewerbs bezwecken oder bewirken, sind verboten."

53 Ein nützlicher Vergleich und wie mir scheint eine zutreffende Beurteilung der unterschiedlichen ordnungs- und wettbewerbspolitischen Vorstellungen Euckens und Hayeks findet sich bei Kolev (2013), S. $63 \mathrm{ff}$.

\section{Literaturverzeichnis}

Aghion, Phillipe und Peter Howitt (1992), A model of growth through creative destruction, Econometrica, Vol. 60, S. 323-351.

Aghion, Philippe und Peter Howitt (2009), The Economics of Growth, Cambridge.

Armentano, Dominick T. (1990), Antitrust and Monopoly: Anatomy of a Policy Failure, 2. Auflage. Oakland.
Doch beging er nicht den Fehler der Ordoliberalen um Eucken und Böhm, für die es Ziel der Wirtschaftspolitik war, die Welt der vollständigen Konkurrenz von Kleinbetrieben als die einzige konsumentenfreundliche und gerechte herzustellen. Eine solche Welt nützte dem Konsumenten keineswegs und würde keinen Wohlstand schaffen. Im Gegenteil: In demselben Maß wie sie Gleichheit unter den Produzenten herstellt, entmachtet sie den Konsumenten und verhindert wohlstandsschaffende Innovation. Nach der Eliminierung aller Marktmacht, nach Erreichen des angeblich idealen "Zustands allseitiger Machtlosigkeit“ (Böhm 1947/2007, S. 69), bliebe allein die Macht des Staates und seiner Behörden, die darüber entschieden, was dem Konsumenten nützt. Dieser wäre damit entmündigt. Wohlstand für alle jedoch kommt nicht durch Entmündigung der Bürger zustande - ein Fazit, dem Erhard sicher zustimmen würde.

Erhards Konzept der allgemeinen Wohlstandsmehrung durch Markt und Wettbewerb ist auch heute noch der beste Gegenentwurf zu den Verirrungen sozialstaatlichen Denkens und politischen Agierens. Die Behauptung, wir verdankten unseren Wohlstand dem Sozialstaat, der Umverteilung, gewerkschaftlichem Druck auf die Löhne, der Sozialpartnerschaft usw. entspringt einer Fehlüberlegung, welche die wahren Ursachen von Wohlstand übersieht: unternehmerisches Handeln, Innovation, technologischer Fortschritt und damit verbundene Produktivitätssteigerung. Die falsch verstandene soziale Marktwirtschaft mag zu einer kurzfristigen Entschärfung sozialer Konflikte geführt haben; mehr Wohlstand konnte sie nicht schaffen. Dafür müssen wir - und die kommenden Generationen - jetzt aber den Preis bezahlen: Mangelndes Wachstum, öffentliche Überschuldung, Druck auf die Reallöhne, schleichende Enteignung des Sparers, kurz: schwindender Wohlstand. Politiker, Zentralbanker und sozial eingestellte Kritiker des freien Marktes preisen nun aber als Heilmittel dagegen genau das an, was all diese Probleme verursacht hat. Genau deshalb ist Erhards Konzeption Wohlstand durch Wettbewerb auch heute noch von höchster Aktualität. Sie weist, nicht zuletzt auch mit ihren moralischen Impulsen, den Weg in die richtige Richtung.
Armentano, Dominick T. (2007), Antitrust. The Case for Repeal, 2. überarbeitete Auflage, Auburn.

Blaich, Fritz (1970), Die Anfänge der deutschen Antikartellpolitik zwischen 1897 und 1914, Jahrbuch für Sozialwissenschaft, Bd. 21, S. 127-150.

Blankart, Charles B. (2011), Öffentliche Finanzen in der Demokratie. Eine Einführung in die 
Finanzwissenschaft, 8. vollständig überarbeitete Auflage, München.

Böhm, Franz (1933/2010) Wettbewerb und Monopolkampf, 1. Neuausgabe, Baden-Baden.

Böhm, Franz (1947/2007), Kartellauflösung und Konzernentflechtung. Spezialistenaufgabe oder Schicksalsfrage?, in: Roser Traugott und Walter Oswalt (Hg.) (2007), Entmachtung durch Wettbewerb, Berlin, S. 59-91.

Boldrin, Michele und David K. Levine (2008), Against Intellectual Monopoly, Cambridge und New York.

Bracher, Karl Dietrich (1971), Die Auflösung der Weimarer Republik. Eine Studie zum Problem des Machtverfalls in der Demokratie, 5. Auflage, Villingen.

Briefs, Goetz (1966), Staat und Wirtschaft im Zeitalter der Interessenverbände, in: Goetz Briefs (Hrsg.), Laissez-faire-Pluralismus. Demokratie und Wirtschaft des gegenwärtigen Zeitalters, Berlin, S. 1-317.

Bundeskartellamt (2014), Erste Bußgelder im Kartellverfahren gegen Bierbrauer verhängt, URL: http:// www.bundeskartellamt.de/SharedDocs/Meldung/ DE/Pressemitteilungen/2014/13_01_2014_Fernsehbiere.html (abgerufen am 03.03.2017).

Commun, P. (2015), Die Ordoliberalen: Vordenker von Erhards Sozialer Marktwirtschaft oder Waffenbrüder? Anmerkungen zum Buch von Horst Friedrich Wünsche, ORDO, Vol. 66, S. 311-316.

Diamond, Peter, A. (1965), National Debt in a Neoclassical Growth Model, The American Economic Review, Vol. 55, S. 1126-1150.

Diamond, Peter A. und Peter R. Orszag (2005), Saving Social Security: A Balanced Approach, Washington D.C.

Dörr, J. (2015), Der verkannte Ludwig Erhard? Anmerkungen zum Buch von Horst Friedrich Wünsche, ORDO, Vol. 66, 303-310.

Eickhof, Norbert (2009), Die Hoppmann-Kantzenbach-Kontoverse aus heutiger Sicht, in: Viktor J. Vanberg (Hg.), Evolution und freiheitlicher Wettbewerb. Erich Hoppmann und die aktuelle Diskussion, Tübingen, S. 35-59.

Erhard, Ludwig (1949/1988), Kartelle im Blickpunkt der Wirtschaftspolitik, in Karl Hohmann (Hg.) (1988), Gedanken aus fünf Jahrzehnten. Reden und Schriften. Düsseldorf, Wien, New York, S. 217-223.

Erhard, Ludwig, (1955/1988), Die Ziele des Gesetzes gegen Wettbewerbsbeschränkungen, in: Karl Hohmann (Hg.) (1988), Gedanken aus fünf Jahrzehnten. Reden und Schriften. Düsseldorf, Wien und New York, S. 430-439.

Erhard, Ludwig (1964), Wohlstand für alle. Bearbeitet von Wolfram Langer, 8. Auflage, Düsseldorf.
Erhard, Ludwig (1977), Erbe und Auftrag. Aussagen und Zeugnisse, Düsseldorf, Wien.

Erhard, Ludwig (1964/1988), Franz Oppenheimer, dem Lehrer und Freund. Rede zu Oppenheimers 100. Geburtstag in der Freien Universität Berlin, 30. April 1964, in: Karl Hohmann (Hg.) (1988), Gedanken aus fünf Jahrzehnten. Reden und Schriften. Düsseldorf, Wien und New York, S. 858-864.

Erhard. Ludwig (1971/1988), Das Ordnungsdenken der Marktwirtschaft. Festschrift zum 90. Geburtstag von Ludwig von Mises, in: Karl Hohmann (Hg.) (1988), Gedanken aus fünf Jahrzehnten. Reden und Schriften. Düsseldorf, Wien und New York, S. 1040-1052.

Erhard, Ludwig und Alfred Müller-Armack (1972), Soziale Marktwirtschaft. Ordnung der Zukunft. Manifest '72, Frankfurt/M, Berlin und Wien.

Eucken, Walter (1947/2012), Überlegungen zum Monopolproblem, in: Walter-Eucken-Archiv (Hg.) (2012), Wirtschaftsmacht und Wirtschaftsordnung, Berlin, S. 79-84.

Eucken, Walter (1950/2012), Vorträge zur Wirtschaftspolitik, 1. Vortrag: Das Problem der wirtschaftlichen Macht, in: Walter-Eucken-Archiv (Hg.) (2012), Wirtschaftsmacht und Wirtschaftsordnung, Berlin, S. 9-22.

Eucken, Walter (1952), Grundsätze der Wirtschaftspolitik, Tübingen.

Frey, Bruno S. und Gebhard Kirchgässner (1994), Demokratische Wirtschaftspolitik. Theorie und Anwendung, 2. Völlig neubearbeitete Auflage, München.

Giersch, Herbert (1961), Allgemeine Wirtschaftspolitik. Grundlagen, Wiesbaden.

Grossman, Gene M. und Elhanan Helpman (1994), Endogenous Innovation in the Theory of Growth, Journal of Economic Perspectives, Vol. 8, S. 23-44.

Haselbach, Dieter (1985), Franz Oppenheimer: Soziologie, Geschichtsphilosophie und Politik des „Liberalen Sozialismus“, Opladen.

Hayek, Friedrich A. von (1945/2004), Der Weg zur Knechtschaft, Tübingen.

Hayek, Friedrich A. von (1946/2003), Der Sinn des Wettbewerbs, in: Friedrich A. von Hayek (2003), Rechtsordnung und Handelsordnung. Aufsätze zur Ordnungsökonomik, Tübingen, S. 107-120.

Hayek, Friedrich A. von (1968/2003), Der Wettbewerb als Entdeckungsverfahren, in: Friedrich $A$. von Hayek (2003), Rechtsordnung und Handelsordnung. Aufsätze zur Ordnungsökonomik, Tübingen,132-149.

Hayek, Friedrich A. von (2003), Recht, Gesetz und Freiheit. Eine Neufassung der liberalen Grundsätze 
der Gerechtigkeit und der politischen Ökonomie, Tübingen.

Helpman, Elhanan (2004), The Mystery of Economic Growth, Cambridge, London.

Heuss, Ernst (1965), Allgemeine Markttheorie, Tübingen.

Hidy, Ralph und Muriel Hidy (1955), Pioneering in Big Business 1882-1911: History of the Standard Oil Company (New Jersey), New York.

Holtfrerich, Carl-Ludwig, Lars P. Feld und Werner Heun et al. (2015): Staatsschulden: Ursachen, Wirkungen und Grenzen, Berlin.

Hoppmann, Erich (1968), Warum Wettbewerbspolitik?, Die Aussprache, Vol. 18, S. 165-169.

Hoppmann, Erich (1972), Fusionskontrolle, Tübingen.

Hoppmann, Erich (1977), Marktmacht und Wettbewerb, Tübingen.

Jones, Charles I. und Paul Romer (2009), The New Kaldor Facts: Ideas, Institutions, Population, and Human Capital, NBER Workings Paper 15094, National Bureau of Economic Research, Cambridge.

Josephson, Matthew (1934/1995), The Robber Barons, San Diego, New York und London.

Kealey, Terence (1996), The Economic Laws of Scientific Research, Houndmills, London und New York.

Kirzner, Israel M. (1973), Competition and Entrepreneurship, Chicago.

Kolev, Stefan (2013), Neoliberale Staatverständnisse im Vergleich, Stuttgart.

Kolko, Gabriel (1963/1977), The Triumph of Conservatism. A Reinterpretation of American History, 19001916, New York.

Kolko, Gabriel (2012), The New Deal Illusion, URL: http://www.counterpunch.org/2012/08/29/the-newdeal-illusion/ (abgerufen im September 2016).

Kurzlechner, Werner (2008), Fusionen, Kartelle, Skandale. Das Bundeskartellamt als Wettbewerbshüter und Verbraucheranwalt, München.

Lenel, Hans Otto (1975): Vollständiger und freier Wettbewerb als Leitbilder für eine Wettbewerbspolitik gegenüber mächtigen Unternehmen, in: Sauermann, Heinz und Ernst-Joachim Mestmäcker (Hg.), Wirtschaftsordnung und Staatsverfassung. Festschrift für Franz Böhm zum 80. Geburtstag, Tübingen, S. 317-340.

Mantzavinos, C. (2001), Individuals, Institutions, and Markets, Cambridge.

Mantzavinos, C. (2006), The institutional-evolutionary antitrust model, European Journal of Law and Economics, Vol. 22, S. 273-291.
Mazzucato, Mariana (2014), Das Kapital des Staates. Eine andere Geschichte von Innovation und Wachstum, München.

Mingardi, Alberto (2015), A Critique of Mazzucato's Entrepreneurial State, Cato Journal, Vol. 35, S. 603-625.

Mises, Ludwig von (1929/1976), Kritik des Interventionismus. Untersuchungen zur Wirtschaftspolitik und Wirtschaftsideologie der Gegenwart, Jena 1929; Neuauflage, Darmstadt.

Mises, Ludwig von (1932), Die Legende vom Versagen des Kapitalismus, in: S.V. Kardoff, H. Schäfer, G. Briefs und $\mathrm{H}$. Kroner (Hg.), Der internationale Kapitalismus und die Krise. Festschrift für Julius Wolf, Stuttgart, S. 23-29.

Mises, Ludwig von (1940/2010), Nationalökonomie, Theorie des Handelns und Wirtschaftens, Genf.

Mises, Ludwig von (1949/1998), Human Action. A Treatise on Economics, Auburn.

Müller-Armack, Alfred (1956/1976), Soziale Marktwirtschaft, in: Müller-Armack (1976), Wirtschaftsordnung und Wirtschaftspolitik. Studien und Konzepte zur Sozialen Marktwirtschaft und zur Europäischen Integration, 2. Auflage, Bern und Stuttgart, S. 243-249.

Müller-Armack, Alfred (1974), Genealogie der Sozialen Marktwirtschaft. Frühschriften und weiterführende Konzepte, Bern und Stuttgart.

Müller-Armack, Alfred (1990), Wirtschaftslenkung und Marktwirtschaft, Sonderausgabe München.

Nell-Breuning, Oswald von SJ. (1986/1990), Wie „sozial“ ist die „Soziale Marktwirtschaft", in: Friedhelm Hengsbach SJ, Den Kapitalismus umbiegen. Schriften zur Kirche, Wirtschaft und Gesellschaft, Düsseldorf, S. 222-238.

n-tv (2014), Brauereien klüngeln Preise aus. Bierbrauer müssen Millionen-Strafe zahlen, URL: http://www.n-tv.de/wirtschaft/Millionenstrafe-fuerBierbrauer-Kartellamt-ahndet-Preisabsprachenarticle12066986.html (abgerufen am 03.03.2017).

Olson, Mancur (1965/2004), Die Logik des kollektiven Handelns: Kollektivgüter und die Theorie der Gruppen, 5. Auflage, Tübingen.

Olson, Mancur (1982/1991), Aufstieg und Niedergang von Nationen. Ökonomisches Wachstum, Stagflation und soziale Starrheit, 2. Auflage, Tübingen.

Oppenheimer, Franz (1938/1998), Das Kapital. Kritik der politischen Ökonomie, in: Julius H. Schoeps, Alphons Silbermann, Hans Süssmuth in Verbindung mit Bernhard Vogt, Gesammelte Schriften, Band III: Schriften zur Marktwirtschaft, Berlin, S. 449-801. 
Pigou, A. C. (1932/2013), Economics of Welfare, Fourth edition (First edition 1920), Basingstoke und New York.

Ridley, Matt (2015), The Evolution of Everything: How Small Changes Transform Our World, London.

Romer, Paul (1986), Increasing returns and long-run growth, Journal of Political Economy, Vol. 94, 1002-1037.

Romer, Paul (1990), Endogenous technological change, Journal of Political Economy, Vol. 98, 71-102.

Röpke, Wilhelm (1950), Ist die deutsche Wirtschaftspolitik richtig? Analyse und Kritik, Stuttgart und Köln

Rüstow, Alexander (1945/2001), Das Versagen des Wirtschaftsliberalismus, 3.überarbeitete Auflage, Marburg.

Samuelson, Paul A. und William D. Nordhaus (1998), Economics - Volkswirtschaftslehre. Übersetzung der 15. amerikanischen Ausgabe. Unter Mitarbeit von Michael J. Mandel, Wien und Frankfurt 1998.

Schmidt, Ingo und Justus Haucap (2013), Wettbewerbspolitik und Kartellrecht. Eine interdisziplinäre Einführung, 10. Auflage, München.

Schumpeter, Joseph A. (1946), Kapitalismus, Sozialismus und Demokratie, Bern.

Shapiro, Daniel (2007), Is the Welfare State Justified? Cambridge, New York etc.

Stamate-Ștefan, Andreas und Mihai Vladimir Topan (2015), The Economic Theory of European
Competition Policy. Retrospectives and Perspectives, Athens: ATINER'S Conference Paper Series, No: CBC2014-1361.

Tarbell, Ida M. (1966), The History of the Standard Oil Company. Briefer Version, New York.

Vogt, Bernhard (1999), Franz Oppenheimer und die Grundlegung der sozialen Marktwirtschaft, in: Elke-Vera Kotowski, Julius H. Schoeps und Bernhard Vogt (Hg.), Wirtschaft und Gesellschaft. Franz Oppenheimer und die Grundlegung der sozialen Marktwirtschaft, Berlin und Bodenheim, S. 243-262.

Wagenknecht, Sahra (2016), Reichtum ohne Gier. Wie wir uns vor dem Kapitalismus retten, Frankfurt und New York.

Walter-Eucken-Archiv (2012), Wirtschaftsmacht und Wirtschaftsordnung, Berlin.

Warsh, David (2006), Knowledge and the Wealth of Nations. A Story of Economic Discovery, New York und London.

White, Richard (2011), Railroaded. The Transcontinentals and the Making of Modern America, New York.

Witt, Ulrich (1987), Individualistische Grundlagen der evolutorischen Ökonomik, Tübingen.

Wünsche, Horst Friedrich (2015), Ludwig Erhards Soziale Marktwirtschaft. Wissenschaftliche Grundlagen und politische Fehldeutungen, Reinbek. 